\title{
AN X-RAY AND INFRARED SURVEY OF THE LYNDS 1228 CLOUD CORE
}

\author{
STEPHen L. SkinNeR ${ }^{1}$, Luisa RebUlL ${ }^{2}$, AND MANUel GÜDEL ${ }^{3}$ \\ ${ }^{1}$ CASA, University of Colorado, Boulder, CO 80309-0389, USA; stephen.skinner@ colorado.edu \\ ${ }^{2}$ Spitzer Science Center/Caltech, M/S 220-6, 1200 East California Blvd., Pasadena, CA 91125, USA; rebull@ipac.caltech.edu \\ ${ }^{3}$ Department of Astrophysics, University of Vienna, Türkenschanzstr. 17, A-1180 Vienna, Austria; manuel.guedel@univie.ac.at \\ Received 2013 November 1; accepted 2014 January 13; published 2014 March 14
}

\begin{abstract}
The nearby Lynds 1228 (L1228) dark cloud at a distance of $\sim 200 \mathrm{pc}$ is known to harbor several young stars including the driving sources of the giant HH 199 and HH 200 Herbig-Haro (HH) outflows. L1228 has previously been studied at optical, infrared, and radio wavelengths but not in X-rays. We present results of a sensitive $37 \mathrm{ks}$ Chandra ACIS-I X-ray observation of the L1228 core region. Chandra detected $60 \mathrm{X}$-ray sources, most of which are faint ( $<40$ counts) and non-variable. Infrared counterparts were identified for 53 of the $60 \mathrm{X}$-ray sources using archival data from the Two Micron All-Sky Survey, the Spitzer Space Telescope, and the Wide-field Infrared Survey Explorer. Object classes were assigned using mid-IR colors for those objects with complete photometry, most of which were found to have colors consistent with extragalactic background sources. Seven young stellar object candidates were identified including the class I protostar HH 200-IRS which was detected as a faint hard X-ray source. No X-ray emission was detected from the luminous protostar HH 199-IRS. We summarize the X-ray and infrared properties of the detected sources and provide IR spectral energy distribution modeling of high-interest objects including the protostars driving the HH outflows.
\end{abstract}

Key words: Herbig-Haro objects - ISM: clouds - stars: formation - X-rays: stars

Online-only material: color figures, supplemental data

\section{INTRODUCTION}

Lynds 1228 (hereafter L1228; centered at J2000 R.A. = $20^{\mathrm{h}} .97$, decl. $=+77^{\circ} .32$ ) is one of the closest dark clouds in the catalog of Lynds (1962). It is part of the Cepheus Flare region which in its entirety comprises one of the nearest giant molecular clouds. The Cepheus Flare consists of as many as eight different dark cloud complexes widely distributed over $\sim 100 \mathrm{deg}^{2}$ (Kun 1998; Kiss et al. 2006). The main cloud complex lies at $d=$ $300 \pm 30 \mathrm{pc}$ but L1228 is in the foreground at $d=200_{-20}^{+100} \mathrm{pc}$ (Kun 1998; Kun et al. 2008). The total mass of L1228 is estimated to be $M_{\text {cloud }} \sim 230 M_{\odot}$ which is intermediate within the range $\sim 40-1500 M_{\odot}$ for the other cloud complexes in the Cepheus Flare (Kirk et al. 2009).

Star-formation has occurred in several of the clouds as evidenced by the discovery of a number of low-mass young stellar objects (YSOs) in various surveys, as summarized below. But several dense cores are also present which appear starless and may eventually form low-mass stars (Kun 1998). Starformation in some of the clouds may have been triggered by a supernova that exploded $\sim 40,000 \mathrm{yr}$ ago whose vestige is an expanding hot bubble known as the Cepheus Flare Shell (Grenier et al. 1989; Olano et al. 2006). L1228 is of particular interest since its projected position lies just inside the current radius of this expanding shell (Figure 1 of Kirk et al. 2009). As such, it emerges as a likely candidate for recent star-formation.

An objective prism survey of L1228 was undertaken by Ogura \& Sato (1990) to identify candidate pre-main sequence (PMS) stars. Their survey identified $69 \mathrm{H} \alpha$ emission stars, 9 of which clustered near the central part of L1228. Using optical spectroscopy and photometry in combination with Two Micron All-Sky Survey (2MASS) near-IR and Spitzer Space Telescope Infrared Array Camera (IRAC) and MIPS-24 $\mu \mathrm{m}$ data, Kun et al. (2009) identified 13 PMS stars in L1228 with extinctions in the range $A_{\mathrm{V}}=0.0-5.9 \mathrm{mag}$ (median $A_{\mathrm{V}} \approx 3 \mathrm{mag}$ ) and ages spanning 1-10 Myr with a median of $\sim 3 \mathrm{Myr}$. The Spitzer Gould Belt Survey (Kirk et al. 2009) included L1228 in which 23 YSO candidates were identified. Further analysis of $J H K$ photometry combined with Spitzer IRAC and Multiband Imaging Photometer for Spitzer (MIPS) $24 \mu \mathrm{m} / 70 \mu \mathrm{m}$ data by Chapman \& Mundy (2009) identified seven YSOs in the L1228 core region. The YSOs identified so far are predominantly class II objects (e.g., Figure 8 of Kirk et al. 2009), also known as classical T Tauri stars (cTTSs). These objects are low-mass PMS stars still surrounded by accretion disks. However, at least two young class I protostars are also present, HH 199-IRS and HH 200-IRS. High-mass stars such as the young OB stars found in Orion are not currently present and the Cepheus Flare complex thus more closely resembles regions such as Taurus where low-mass stars are the rule.

The studies cited above have focused on optical and IR identification of candidate YSOs. X-ray observations are also useful for this purpose since low-mass YSOs typically show elevated levels of $\mathrm{X}$-ray emission relative to their main sequence counterparts. L1228 has not previously been observed in a pointed X-ray observation but it was part of the ROSAT AllSky Survey (RASS) coverage region in Cepheus studied by Tachihara et al. (2005). They identified 16 weak-lined T Tauri stars (wTTSs) in Cepheus but none lie in L1228. We present here the results of a more sensitive X-ray observation of the L1228 cloud core with Chandra. Our goals are to obtain the first census of X-ray sources in the cloud core and identify potential X-ray emitting YSOs. Because of the proximity of L1228 and its relatively low extinction, the observation provides high sensitivity and is capable of detecting X-ray emitting TTSs down to the low end of the stellar mass limit (Section 2). We also analyze IR data from 2MASS, Spitzer, and the Wide-field Infrared Survey Explorer (WISE) in order to identify counterparts to X-ray sources, distinguish between YSOs and extragalactic contaminants, and determine basic YSO 
Table 1

Chandra Observation of L1228

\begin{tabular}{lc}
\hline \hline Parameter & Value \\
\hline ObsId & 7425 \\
Start date/time (TT) & 2007 Jul 22/20:33:39 \\
Stop date/time (TT) & 2007 Jul 23/07:13:40 \\
Instrument & ACIS-I \\
Field-of-view (arcmin) ${ }^{\text {a }}$ & $16.9 \times 16.9$ \\
Livetime (s) & 36,779 \\
Frame time (s) & 3.2 \\
\hline
\end{tabular}

Note. a Nominal pointing position was at (J2000) R.A. $=20^{\mathrm{h}} \quad 57^{\mathrm{m}} \quad 02.71$ decl. $=+77^{\circ} \quad 35^{\prime} \quad 45^{\prime \prime} .1$, or $(l, b)=(111.664,+20.226)$.

properties. The study goes beyond previous work by including new X-ray data and by incorporating all previous Spitzer archive data as well as new WISE mid-IR data.

\section{OBSERVATIONS AND DATA REDUCTION}

\subsection{Chandra}

The Chandra observation is summarized in Table 1. The exposure was obtained using the ACIS-I (Advanced CCD Imaging Spectrometer) imaging array in faint timed-event mode. ACIS-I has a combined field of view $(\mathrm{FoV})$ of $\approx 16^{\prime} .9 \times 16^{\prime} .9$ consisting of four front-illuminated $1024 \times 1024$ pixel CCDs with a pixel size of 0.492 . Approximately $90 \%$ of the encircled energy at $1.49 \mathrm{keV}$ lies within $\approx 1^{\prime \prime}$ of the central pixel of an on-axis point source. More information on Chandra and its instrumentation can be found in the Chandra Proposer's Observatory Guide (POG). ${ }^{4}$

The Level 1 events file provided by the Chandra X-ray Center (CXC) was processed using Chandra Interactive Analysis of Observations (CIAO) version $4.4^{5}$ standard science threads. The CIAO processing script chandra_repro applied recent calibration updates. We used the CIAO wavdetect tool to identify $\mathrm{X}$-ray sources on the ACIS-I array along with their centroid positions and $3 \sigma$ position error ellipses. Wavdetect was executed on full-resolution images using events in the $0.3-7 \mathrm{keV}$ range to reduce the background. Wavelet radii as set by the scales input parameter were 1, 2, 4, 8, and 16 pixels. Background was very low, amounting to 0.0104 counts per pixel $(0.3-7 \mathrm{keV})$ integrated over the full $36.8 \mathrm{ks}$ exposure. A nominal on-axis source extraction region of radius $r=1^{\prime \prime}$ contains only $\approx 0.14$ background counts $(0.3-7 \mathrm{keV})$. We retained only those X-ray sources found by wavdetect having $\geqslant 5$ net counts in our final source list. This threshold corresponds to an unabsorbed X-ray luminosity detection limit $L_{x} \approx 10^{28.05} \mathrm{erg} \mathrm{s}^{-1}(0.3-7 \mathrm{keV})$ at $d=200 \mathrm{pc}$, assuming a generic TTS thermal X-ray spectrum with plasma temperature $k T=3 \mathrm{keV}$ and hydrogen absorption column density $N_{\mathrm{H}}=10^{21.8} \mathrm{~cm}^{-2}\left(A_{\mathrm{V}} \approx 3 \mathrm{mag}\right)$. This extinction is typical for the cloud as a whole (Kun et al. 2009) but $A_{\mathrm{V}}$ is higher toward the most obscured central regions (Kiss et al. 2006; Chapman \& Mundy 2009; Kirk et al. 2009). Using the known correlations between $L_{x}$ and stellar mass $\left(M_{*}\right)$ for TTS as determined from deep Chandra survey of the Orion Nebula Cluster (Preibisch et al. 2005), and from the XMM-Newton survey of the Taurus Molecular Cloud (Telleschi et al. 2007),

\footnotetext{
4 See http://asc.harvard.edu/proposer/POG

5 Further information on Chandra Interactive Analysis of Observations (CIAO) software can be found at http://asc.harvard.edu/ciao
}

the above $L_{x}$ limit corresponds to a TTS of mass $M_{*} \lesssim 0.1 M_{\odot}$. The CIAO tool specextract was used to extract X-ray spectra of selected bright sources and spectral fitting was carried out with HEASOFT XSPEC software version 12.4.0. ${ }^{6}$ Source variability probabilities were computed using the CIAO tool glvary which is based on the Gregory-Loredo algorithm (Gregory \& Loredo 1992, 1996).

\subsection{Infrared Archive Data (2MASS, Spitzer, WISE)}

We have used existing IR archive data to identify potential IR counterparts to X-ray sources, obtain IR photometry of highinterest sources, and determine object classes in those cases where good-quality multi-band photometry are available.

2MASS. We obtained near-IR positions and magnitudes from the 2MASS All-Sky Catalog (Skrutskie et al. 2006). 2MASS provides photometry at $J(1.25 \mu \mathrm{m}), H(1.65 \mu \mathrm{m})$, and $K_{S}$ $(2.17 \mu \mathrm{m})$. The spatial resolution of $\approx 1^{\prime \prime} .2$ is comparable to that of Chandra and to Spitzer at $3.6 \mu \mathrm{m}$.

Spitzer. The Spitzer Space Telescope (Werner et al. 2004) observed the L1228 region multiple times with the IRAC (Fazio et al. 2004) and the MIPS (Rieke et al. 2004). IRAC provides data in four channels: $I 1(3.6 \mu \mathrm{m}), I 2(4.5 \mu \mathrm{m}), I 3(5.8 \mu \mathrm{m})$, and $I 4(8.0 \mu \mathrm{m})$. The IRAC native pixel size is $1^{\prime \prime} .2$ with spatial resolution FWHM $\approx 1$ 1. $6-2$ '. 0 . MIPS has three channels: MIPS-24 (24 $\mu \mathrm{m})$, MIPS-70 (70 $\mu \mathrm{m})$, and MIPS-160 $(160 \mu \mathrm{m})$ with respective spatial resolutions FWHM $\approx 6^{\prime \prime}, 18^{\prime \prime}$, and $40^{\prime \prime}$. Further information on these instruments can be found in the respective Instrument Handbooks. ${ }^{7}$ We have reprocessed all Spitzer IRAC and MIPS-24 archive data (Table 2) intersecting the Chandra FoV to generate deep-coverage image mosaics. Since MIPS-70 and MIPS-160 band data are not available for all the MIPS Astronomical Observation Requests (AORs) we have only reprocessed the MIPS-24 data. However, MIPS-70 photometry are available for some sources such as HH 199-IRS and HH 200-IRS from the Cores-to-Disks (c2d) Spitzer Legacy program (Evans et al. 2003). We reprocessed the AORs starting from the IRAC pipeline-corrected basic calibrated data (BCD) files or, for MIPS photometry observations, the enhanced BCD (eBCD) files. For MIPS scan maps we started from the BCD files since eBCD files are not available. We used MOPEX (Makovoz \& Marleau 2005) to calculate overlap corrections and to create exposure-time-weighted mosaics with a substantial reduction in instrumental artifacts. Exposure time weighting is necessary since the total integration time varies as a function of position on the sky. The pixel size for our mosaics was the same as the pipeline mosaics, 0'.6 for IRAC and 2".45 for MIPS-24. The Spitzer mosaics cover the full ACIS-I FoV except for the NE and SW corners where $2 \mathrm{MASS}$ and WISE provide coverage. We used the IDL aper.pro routine to perform aperture photometry at the IR peak positions of sources of interest. For the IRAC mosaics we used an aperture radius $r=3$ ". 6 and a background annulus of $r=3$ ". $6-8$ ". 4 . Aperture corrections in the four IRAC channels $(1.124,1.127,1.143$, and 1.234 respectively) and IRAC zero-point magnitudes (280.9, 179.7, 115.0, and 64.13 Jy, respectively) were taken from the IRAC Instrument Handbook. Point-response function (PRF) fitting of MIPS-24 sources was carried out with MOPEX. In those cases where PRF fitting was not successful we obtained MIPS-24 aperture photometry using an aperture of radius $r=7^{\prime \prime}$, an annulus of $r=7^{\prime \prime}-13^{\prime \prime}$,

\footnotetext{
6 http://heasarc.gsfc.nasa.gov/docs/xanadu/xanadu.html

7 http://irsa.ipac.caltech.edu/data/SPITZER/docs/
} 
Table 2

Spitzer Observations of L1228

\begin{tabular}{|c|c|c|c|c|c|c|}
\hline Instrument & Mode & Program (PI) & AORKEY ${ }^{\mathrm{a}}$ & $\begin{array}{c}\text { Frame Time } \\
\text { (s) }\end{array}$ & $\begin{array}{c}\text { Observation } \\
\text { Date }\end{array}$ & Notes \\
\hline IRAC & Map & 104 (Soifer) & 6577664 & $12^{b}$ & 2003 Dec 6 & First Look Survey (FLS) \\
\hline IRAC & Map & 139 (Evans) & 5161216 & $12^{\mathrm{b}}$ & 2003 Dec 2 & Cores-to-Disks Legacy program \\
\hline IRAC & Map & 3656 (Mundy) & 11400192 & 30 & 2004 Dec 15 & \\
\hline IRAC & Map & 3656 (Mundy) & 11391232 & 30 & 2004 Nov 27 & \\
\hline MIPS & Scan & 104 (Soifer) & 6577408 & 2.6 & 2003 Dec 8 & FLS; MIPS 24, 70, $160 \mu \mathrm{m}$ \\
\hline MIPS & Photom. & 139 (Evans) & 5161472 & 2.6 & 2003 Dec 13 & Very small footprint \\
\hline MIPS & Photom. & 3656 (Mundy) & 11397632 & 9.96 & 2004 Dec 26 & MIPS $24 \mu \mathrm{m}$ only \\
\hline MIPS & Photom. & 3656 (Mundy) & 11395072 & 9.96 & $2004 \operatorname{Dec} 7$ & MIPS $24 \mu \mathrm{m}$ only \\
\hline
\end{tabular}

Notes.

a An Astronomical Observation Request (AOR) is the fundamental unit of Spitzer observing. An AORKEY is the unique eight-digit identifier for the AOR, which can be used to retrieve these data from the Spitzer archive.

${ }^{\mathrm{b}}$ Obtained in high dynamic range (HDR) mode, consisting of a short $0.6 \mathrm{~s}$ and long $12 \mathrm{~s}$ frame at each pointing.

and an aperture correction of 2.05 as per the MIPS Instrument Handbook.

WISE. We searched for mid-IR counterparts of X-ray sources using the 2012 March 14 release of the WISE All-Sky Catalog (Wright et al. 2010). WISE completed an all-sky survey during its cryogenic mission phase from 2010 January to August. WISE provides photometry in four bands: $W 1(3.4 \mu \mathrm{m}), W 2(4.6 \mu \mathrm{m})$, $W 3(12 \mu \mathrm{m})$, and $W 4(22 \mu \mathrm{m})$. Time variability can also be assessed based on comparison of fluxes in each band for a given source obtained at different times during the mission. WISE sensitivity is somewhat less than obtained in our mosaicked Spitzer images of L1228 and WISE spatial resolution is modest with FWHM values of 6.' 1,6 , 4,6 ".5, and $12^{\prime \prime} .0$ in the $W 1, W 2, W 3$, W4 bands. WISE images and associated data in all four bands (e.g., instrumental profile-fit photometry) were downloaded from the archive for analysis.

\section{X-RAY RESULTS}

\subsection{Source Identification}

Chandra detected $60 \mathrm{X}$-ray sources on ACIS-I with $\geqslant 5$ net counts. Their positions on the ACIS-I detector are plotted in Figure 1 and source properties are summarized in Table 3. Most of the X-ray sources are faint and only 8 sources have $>50$ net counts. The mean number of counts is 25 and the median is 13 counts. None of the X-ray sources are listed in the less sensitive RASS compilation of Tachihara et al. (2005). We found potential IR counterparts for 53 of the 60 sources (Table 4). In almost all cases the offsets between the X-ray and IR positions are $<2$ ". As Table 4 shows, larger offsets of 2". $0-3$ ". 6 are present for some sources (CXO sources 2, 5, 14, 16, 58, and $60)$. These sources lie off-axis and have large X-ray position error ellipses due to broadening of the point-spread function (PSF), so their IR counterpart identifications given in Table 4 are more uncertain. WISE coverage spans the entire ACIS-I FoV and potential WISE counterparts were found for 30 sources. Four WISE sources have variability flag values varflg $\geqslant 6$ in one or more bands indicating likely IR variability (CXO 26, 27, 30, and 53 ). Figure 2 is an overlay of the X-ray source positions on a WISE W1-band image. The mosaicked Spitzer images are more sensitive than WISE and Spitzer counterparts were found for all but 10 X-ray sources, 7 of which lie outside the Spitzer coverage area (Table 4). IR photometry for each source are summarized in Table 5.

Interestingly, the brightest IR source in the field IRAS $20582+7724=$ HH 199-IRS was not detected by Chandra.

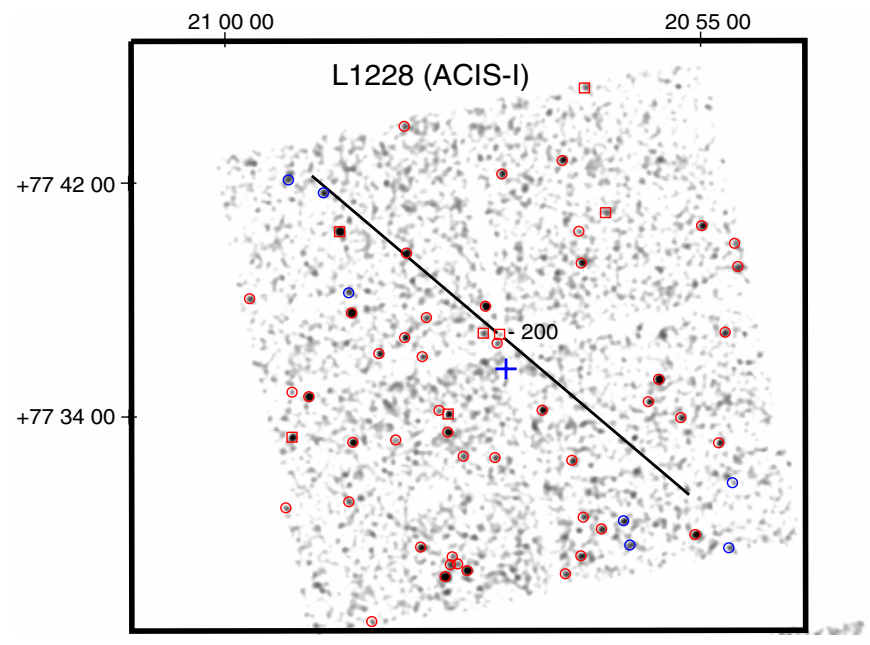

Figure 1. Positions of L1228 X-ray sources overlaid on the Chandra ACIS-I image $(0.3-7 \mathrm{keV})$. The image has been Gaussian-smoothed with a three-pixel kernel. The raw (unsmoothed) pixel size is 0"49. Objects marked in red have candidate IR counterparts (Table 4) and those in blue lack counterparts. Squares denote known or suspected YSOs (Table 3). The object labeled "200" is HH 200-IRS (CXO source nr. 26 in Table 3). The solid lines show the HH 200 bipolar outflow axes at P.A. $=49^{\circ} / 229^{\circ}$, measured east from north (Devine et al. 2009). A cross (+) marks the nominal pointing position. Log intensity scale. J2000 coordinate overlay.

(A color version of this figure is available in the online journal.)

It is a class I protostar that is believed to power the HH 199 outflow (Bally et al. 1995). Its IR properties are discussed further in Section 5. The hardest X-ray source as gauged by its mean photon energy $\bar{E}=5.1 \mathrm{keV}$ is CXO source 26 $(\mathrm{J} 205706.72+773656.1)$. This mean energy is well above the average value $\bar{E}=2.8 \mathrm{keV}$ for the sample (Figure 3 ). The $\mathrm{X}$ ray position of $\mathrm{CXO}$ source 26 as determined by wavdetect is offset by only 0 .'06 from the Very Large Array (VLA) radio source L1228 VLA 4 (Reipurth et al. 2004) which is identified with the IR source HH 200-IRS that is thought to power the HH 200 outflow. As discussed further in Section 5, a second IR source is located 5".7 northeast of HH 200-IRS but is well outside the X-ray position error ellipse and is thus ruled out as the X-ray source. We thus associate this faint hard X-ray source with $\mathrm{HH}$ 200-IRS, a known class I protostar (Chapman \& Mundy 2009). Other previously known YSOs detected by Chandra are CXO sources 30, 36, and 53 and their properties are discussed further in Section 4. 
Table 3

Chandra X-Ray Sources in Lynds $1228^{\mathrm{a}}$

\begin{tabular}{|c|c|c|c|c|c|c|c|c|}
\hline $\begin{array}{l}\mathrm{CXO} \\
\mathrm{Nr} .\end{array}$ & $\begin{array}{c}\text { R.A. } \\
\text { (J2000) }\end{array}$ & $\begin{array}{c}\text { Decl. } \\
\text { (J2000) }\end{array}$ & $\begin{array}{l}\text { Net Counts } \\
\text { (cts) }\end{array}$ & $\begin{array}{c}\bar{E} \\
(\mathrm{keV})\end{array}$ & $P_{\text {var }}$ & IR & IR Class & Notes \\
\hline 1 & 205438.03 & +773913.03 & $11 \pm 4$ & 2.66 & 0.46 & $\mathrm{y}$ & $\ldots$ & 1,2 \\
\hline 2 & 205439.93 & +774000.51 & $9 \pm 4$ & 2.40 & 0.40 & $\mathrm{y}$ & $\ldots$ & 1,2 \\
\hline 3 & 205442.76 & +773149.17 & $7 \pm 3$ & 1.81 & 0.83 & $\mathrm{n}$ & $\ldots$ & \\
\hline 4 & 205445.42 & +772935.74 & $13 \pm 4$ & 2.70 & 0.38 & $\mathrm{n}$ & $\ldots$ & 1 \\
\hline 5 & 205446.34 & +773658.24 & $12 \pm 4$ & 3.65 & 0.47 & $\mathrm{y}$ & $\ldots$ & 2,3 \\
\hline 6 & 205451.05 & +773311.31 & $11 \pm 3$ & 2.65 & 0.60 & $\mathrm{y}$ & AGN & \\
\hline 7 & 205500.39 & +774037.38 & $27 \pm 6$ & 3.12 & 0.55 & $\mathrm{y}$ & AGN & \\
\hline 8 & 205506.20 & +773003.59 & $57 \pm 8$ & 2.38 & 0.96 & $\mathrm{y}$ & AGN & \\
\hline 9 & 205514.38 & +773403.69 & $18 \pm 5$ & 2.42 & 0.36 & $\mathrm{y}$ & AGN & \\
\hline 10 & 205527.94 & +773522.62 & $135 \pm 12$ & 1.29 & 0.99 & $\mathrm{y}$ & none/star & 4 \\
\hline 11 & 205534.40 & +773436.88 & $18 \pm 4$ & 3.20 & 0.55 & $\mathrm{y}$ & $\ldots$ & 2 \\
\hline 12 & 205546.39 & +772942.88 & $7 \pm 3$ & 3.54 & 0.44 & $\mathrm{n}$ & $\ldots$ & \\
\hline 13 & 205550.22 & +773032.87 & $20 \pm 5$ & 2.54 & 0.48 & $\mathrm{n}$ & $\ldots$ & \\
\hline 14 & 205600.44 & +774105.33 & $8 \pm 3$ & 2.46 & 0.43 & $\mathrm{y}$ & galaxy/YSO? & 3,8 \\
\hline 15 & 205603.86 & +773016.28 & $16 \pm 4$ & 3.88 & 0.77 & $\mathrm{y}$ & $\ldots$ & 2 \\
\hline 16 & 205613.39 & +774521.47 & $10 \pm 4$ & 3.77 & 0.67 & $\mathrm{y}$ & YSO (cl. I/II) & 1,3 \\
\hline 17 & 205615.06 & +773040.38 & $7 \pm 3$ & 2.08 & 0.47 & $\mathrm{y}$ & $\ldots$ & 2 \\
\hline 18 & 205615.77 & +773921.97 & $25 \pm 5$ & 1.11 & 0.47 & $\mathrm{y}$ & none & \\
\hline 19 & 205616.58 & +772921.25 & $22 \pm 5$ & 2.52 & 0.35 & $\mathrm{y}$ & $\ldots$ & 2 \\
\hline 20 & 205617.15 & +774027.23 & $7 \pm 3$ & 2.90 & 0.49 & $\mathrm{y}$ & $\ldots$ & 2 \\
\hline 21 & 205621.97 & +773237.25 & $13 \pm 4$ & 3.76 & 0.57 & $\mathrm{y}$ & galaxy/AGN & \\
\hline 22 & 205626.19 & +772844.39 & $10 \pm 3$ & 2.33 & 0.62 & $\mathrm{y}$ & $\ldots$ & 1,2 \\
\hline 23 & 205627.47 & +774252.57 & $21 \pm 5$ & 3.23 & 0.50 & $\mathrm{y}$ & AGN & \\
\hline 24 & 205640.29 & +773420.29 & $27 \pm 5$ & 3.92 & 0.83 & $\mathrm{y}$ & galaxy/AGN & \\
\hline 25 & 205705.32 & +774225.21 & $22 \pm 5$ & 2.71 & 0.50 & $\mathrm{y}$ & galaxy & \\
\hline 26 & 205706.72 & +773656.10 & $5 \pm 2$ & 5.10 & 0.34 & $\mathrm{y}$ & YSO (cl. I) & HH 200-IRS \\
\hline 27 & 205708.23 & +773637.76 & $10 \pm 3$ & 3.57 & 0.33 & $\mathrm{y}$ & AGN & \\
\hline 28 & 205709.55 & +773242.98 & $10 \pm 3$ & 3.64 & 0.60 & $\mathrm{y}$ & none & \\
\hline 29 & 205715.56 & +773753.63 & $81 \pm 9$ & 1.39 & 0.15 & $\mathrm{y}$ & none/star & 5 \\
\hline 30 & 205716.88 & +773658.33 & $12 \pm 3$ & 2.35 & 0.47 & $\mathrm{y}$ & YSO (cl. II) & \\
\hline 31 & 205726.69 & +772850.90 & $57 \pm 8$ & 2.63 & 0.99 & $\mathrm{y}$ & galaxy/AGN & \\
\hline 32 & 205729.17 & +773245.55 & $6 \pm 2$ & 4.48 & 0.33 & $\mathrm{y}$ & AGN & \\
\hline 33 & 205732.51 & +772904.68 & $8 \pm 3$ & 3.25 & 0.44 & $\mathrm{y}$ & $\cdots$ & 2 \\
\hline 34 & 205735.86 & +772919.31 & $9 \pm 3$ & 2.86 & 0.44 & $\mathrm{y}$ & galaxy/AGN & \\
\hline 35 & 205736.97 & +772903.02 & $9 \pm 3$ & 1.99 & 0.47 & y & AGN & \\
\hline 36 & 205738.62 & +773412.32 & $25 \pm 5$ & 1.40 & 0.47 & y & none/YSO & $6 ; \mathrm{H} \alpha$ em. star \\
\hline 37 & 205738.82 & +773334.92 & $40 \pm 6$ & 3.58 & 0.40 & $\mathrm{y}$ & galaxy/AGN & \\
\hline 38 & 205740.24 & +772838.71 & $123 \pm 12$ & 2.38 & 0.59 & $\mathrm{y}$ & AGN & \\
\hline 39 & 205744.26 & +773419.56 & $8 \pm 3$ & 3.99 & 0.48 & $\mathrm{y}$ & AGN & \\
\hline 40 & 205752.30 & +773730.13 & $8 \pm 3$ & 3.56 & 0.65 & $\mathrm{y}$ & AGN & \\
\hline 41 & 205754.81 & +773609.83 & $14 \pm 4$ & 4.05 & 0.39 & y & AGN & \\
\hline 42 & 205755.34 & +772939.00 & $24 \pm 5$ & 2.53 & 0.33 & y & galaxy & \\
\hline 43 & 205804.93 & +773942.30 & $29 \pm 5$ & 2.88 & 0.72 & $\mathrm{y}$ & AGN & \\
\hline 44 & 205805.77 & +773648.77 & $21 \pm 5$ & 2.71 & 0.45 & $\mathrm{y}$ & AGN & \\
\hline 45 & 205806.67 & +77 4402.47 & $9 \pm 4$ & 1.70 & 0.65 & $\mathrm{y}$ & none/star & 1,7 \\
\hline 46 & 205811.03 & +773318.63 & $6 \pm 2$ & 3.29 & 0.53 & $\mathrm{y}$ & none & \\
\hline 47 & 205821.78 & +773615.76 & $17 \pm 4$ & 3.60 & 0.46 & $\mathrm{y}$ & galaxy/AGN & \\
\hline 48 & 205825.18 & +772705.70 & $10 \pm 4$ & 3.06 & 0.38 & $\mathrm{y}$ & AGN & 1 \\
\hline 49 & 205837.61 & +773313.36 & $37 \pm 6$ & 1.25 & 0.51 & $\mathrm{y}$ & none & \\
\hline 50 & 205838.98 & +773738.94 & $127 \pm 11$ & 2.54 & 0.98 & $\mathrm{y}$ & AGN & \\
\hline 51 & 205839.79 & +773111.28 & $10 \pm 4$ & 1.79 & 0.34 & $\mathrm{y}$ & $\ldots$ & 2 \\
\hline 52 & 205840.84 & +773820.07 & $11 \pm 3$ & 2.40 & 0.55 & $\mathrm{n}$ & $\ldots$ & \\
\hline 53 & 205846.78 & +774025.47 & $75 \pm 9$ & 1.96 & 0.09 & $\mathrm{y}$ & YSO (cl. II) & 9; $\mathrm{H} \alpha$ em. star \\
\hline 54 & 205857.09 & +774144.97 & $24 \pm 6$ & 2.80 & 0.38 & $\mathrm{n}$ & $\ldots$ & \\
\hline 55 & 205905.10 & +773446.39 & $69 \pm 9$ & 2.63 & 0.11 & $\mathrm{y}$ & AGN & \\
\hline 56 & 205915.26 & +773322.87 & $30 \pm 6$ & 1.32 & 0.62 & y & YSO (cl. II) & \\
\hline 57 & 205915.51 & +773455.20 & $6 \pm 3$ & 3.75 & 0.40 & $\mathrm{y}$ & $\ldots$ & 2 \\
\hline 58 & 205918.68 & +773057.72 & $14 \pm 4$ & 2.43 & 0.20 & $\mathrm{y}$ & AGN & 1,3 \\
\hline 59 & 205919.10 & +774210.71 & $13 \pm 5$ & 3.63 & 0.62 & $\mathrm{n}$ & $\ldots$ & \\
\hline 60 & 205942.55 & +773806.20 & $19 \pm 5$ & 2.23 & 0.40 & $\mathrm{y}$ & none & 1 \\
\hline
\end{tabular}

Notes. 1. Source lies near the edge of the ACIS-I array and is heavily vignetted. 2. No IR class is assigned due to incomplete IR photometry. 3. IR offset $>2$ ". The IR match is questionable. See Table 4 for IR positions. 4. Optical counterpart HST GSC J205527.93+773522.1. Classified as stellar in the GSC. 5. Optical counterpart HST GSC J205715.70+773753.8. Classified as stellar in the GSC. 6. Optical counterpart is H $\alpha$ emission star nr. 38 in Ogura \& Sato (1990). 7. Star. Tycho J205806.80+774402.44. 8. IR classification is ambiguous. 9. Optical counterpart is $\mathrm{H} \alpha$ emission star nr. 42 in Ogura \& Sato (1990).

${ }^{a} \mathrm{X}$-ray data are from ACIS-I using events in the $0.3-7 \mathrm{keV}$ range. Tabulated quantities are: running source number; X-ray source position (R.A., decl.) from wavdetect based on CXC events list with no additional astrometric refinement; net counts and net counts error obtained from wavdetect accumulated in a 36,799 s exposure, rounded to the nearest integer, background subtracted and PSF-corrected; mean photon energy $\bar{E}$; X-ray variability probability $P_{\text {var }}$ from glvary. A y or $\mathrm{n}$ in the IR column denotes whether a potential IR counterpart was found (see Table 4). IR Class is based on IR color analysis using the IRAC criteria of Gutermuth et al. (2008) and WISE criteria of Koenig et al. (2012). 
Table 4

IR Counterparts to X-Ray Sources in L1228

\begin{tabular}{|c|c|c|c|c|}
\hline CXO Nr. & 2MASS & WISE & Spitzer & Notes \\
\hline 1 & & & J205437.98+773911.7 (1.3) & 3 \\
\hline 2 & & & $\mathrm{~J} 205440.45+774002.7(2.7)$ & 3,6 \\
\hline 3 & & & & 1 \\
\hline 4 & & & & 1 \\
\hline 5 & & & $\mathrm{~J} 205447.00+773656.6(2.7)$ & 2,6 \\
\hline 6 & & J205451.29+773311.4 (0.8) & & 1 \\
\hline 7 & & $\mathrm{~J} 205500.21+774037.2(0.6)$ & $\mathrm{J} 205500.28+774036.8(0.7)$ & \\
\hline 8 & & $\mathrm{~J} 205505.85+773005.1(1.9)$ & & 1 \\
\hline 9 & & $\mathrm{~J} 205514.07+773405.0(1.7)$ & J205514.19+773404.4 (0.9) & 2 \\
\hline 10 & $\mathrm{~J} 205527.94+773522.3(0.3)$ & $\mathrm{J} 205527.89+773522.7(0.2)$ & J205527.98+773522.7 (0.2) & 7 \\
\hline 11 & & & $\mathrm{~J} 205534.17+773437.9(1.2)$ & 7 \\
\hline 12 & & & & 2 \\
\hline 13 & & & & 2 \\
\hline 14 & $\mathrm{~J} 205600.25+774105.4(0.6)$ & J205559.46+774103.6 (3.6) & $\mathrm{J} 205559.57+774103.9$ (3.1) & $5,6,7$ \\
\hline 15 & & & J205603.94+773015.8 (0.6) & 4 \\
\hline 16 & & & J205612.79+774518.4 (3.6) & 6 \\
\hline 17 & & & $\mathrm{~J} 205614.93+773040.8(0.6)$ & \\
\hline 18 & J205615.81+773922.5 (0.5) & $\mathrm{J} 205615.73+773922.2(0.3)$ & J205615.94+773921.9 (0.5) & \\
\hline 19 & & & J205616.45+772921.3 (0.4) & 4 \\
\hline 20 & & & $\mathrm{~J} 205617.21+774028.1(0.9)$ & \\
\hline 21 & & $\mathrm{~J} 205621.51+773237.5(1.5)$ & $\mathrm{J} 205622.03+773237.6(0.4)$ & 7 \\
\hline 22 & & & $\mathrm{~J} 205625.85+772843.4(1.5)$ & 4 \\
\hline 23 & & $\mathrm{~J} 205627.39+774253.1(0.6)$ & $\mathrm{J} 205627.29+774253.0(0.7)$ & \\
\hline 24 & & $\mathrm{~J} 205640.35+773421.1(0.8)$ & $\mathrm{J} 205640.29+773420.1(0.2)$ & \\
\hline 25 & & & $\mathrm{~J} 205705.49+774226.6(1.5)$ & 7 \\
\hline 26 & J205706.55+773655.9 (0.6) & J205707.16+773657.4 (1.9) & $\mathrm{J} 205706.61+773656.0(0.4)$ & $5,8,9$ \\
\hline 27 & & J205708.44+773637.7 (0.7) & $\mathrm{J} 205708.23+773637.8(0.0)$ & 9 \\
\hline 28 & & & J205709.66+773242.8 (0.4) & \\
\hline 29 & $\mathrm{~J} 205715.60+773754.0(0.4)$ & $\mathrm{J} 205715.63+773753.8(0.3)$ & J205715.53+773753.8 (0.2) & \\
\hline 30 & $\mathrm{~J} 205716.97+773658.6(0.4)$ & $\mathrm{J} 205716.98+773658.5(0.4)$ & $\mathrm{J} 205717.02+773658.4(0.5)$ & $5,9,10$ \\
\hline 31 & & $\mathrm{~J} 205726.65+772851.4(0.5)$ & J205726.58+772851.2 (0.5) & \\
\hline 32 & & & J205729.15+773245.4 (0.2) & \\
\hline 33 & & & J205732.72+772904.2 (0.8) & \\
\hline 34 & & $\mathrm{~J} 205735.43+772918.5(1.6)$ & $\mathrm{J} 205735.79+772919.4(0.2)$ & 7 \\
\hline 35 & & J205737.40+772902.3 (1.6) & J205737.15+772902.9 (0.6) & \\
\hline 36 & J205738.73+773412.5 (0.4) & $\mathrm{J} 205738.71+773412.4(0.3)$ & J205738.73+773412.3 (0.4) & 11 \\
\hline 37 & & $\mathrm{~J} 205738.82+773335.1(0.2)$ & $\mathrm{J} 205738.79+773334.8(0.2)$ & \\
\hline 38 & & $\mathrm{~J} 205740.44+772838.8(0.7)$ & J205740.46+772838.9 (0.7) & \\
\hline 39 & & & J205744.35+773419.5 (0.3) & \\
\hline 40 & & & $\mathrm{~J} 205752.26+773730.2(0.2)$ & \\
\hline 41 & & & $\mathrm{~J} 205754.83+773610.3(0.5)$ & \\
\hline 42 & & & $\mathrm{~J} 205755.09+772938.1(1.2)$ & 7 \\
\hline 43 & & & J205804.99+773942.8 (0.5) & \\
\hline 44 & & $\mathrm{~J} 205805.86+773648.5(0.4)$ & J205805.86+773648.8 (0.4) & \\
\hline 45 & $\mathrm{~J} 205806.81+774402.4(0.5)$ & $\mathrm{J} 205806.82+774402.3(0.5)$ & $\mathrm{J} 205806.83+774402.5(0.5)$ & 3 \\
\hline 46 & & & $\mathrm{~J} 205811.25+773318.8(0.7)$ & \\
\hline 47 & & $\mathrm{~J} 205821.91+773616.0(0.5)$ & $\mathrm{J} 205821.70+773616.0(0.4)$ & \\
\hline 48 & & $\mathrm{~J} 205825.16+772706.3(0.6)$ & $\mathrm{J} 205825.28+772705.7(0.3)$ & \\
\hline 49 & J205837.68+773314.0 (0.7) & $\mathrm{J} 205837.72+773313.7(0.5)$ & J205837.62+773314.0 (0.6) & \\
\hline 50 & & $\mathrm{~J} 205839.07+773739.0(0.3)$ & $\mathrm{J} 205839.00+773738.9(0.1)$ & \\
\hline 51 & & & J205839.96+773111.4 (0.6) & \\
\hline 52 & & & & 2 \\
\hline 53 & $\mathrm{~J} 205846.68+774025.6(0.3)$ & $\mathrm{J} 205846.71+774025.6(0.3)$ & $\mathrm{J} 205846.71+774026.0(0.6)$ & $2,9,12$ \\
\hline 54 & & & & 1 \\
\hline 55 & & & $\mathrm{~J} 205905.14+773447.5$ (1.1) & \\
\hline 56 & $\mathrm{~J} 205915.41+773322.8(0.5)$ & J205915.40+773322.8 (0.5) & J205915.37+773322.7 (0.4) & \\
\hline 57 & & & $\mathrm{~J} 205915.42+773455.2(0.3)$ & 4,7 \\
\hline 58 & & $\mathrm{~J} 205919.39+773056.7(2.5)$ & J205919.28+773056.7 (2.2) & 6 \\
\hline 59 & & & & 1 \\
\hline 60 & $\mathrm{~J} 205942.32+773808.3(2.2)$ & $\mathrm{J} 205942.35+773808.1(2.0)$ & & 1,6 \\
\hline
\end{tabular}

Notes. The number in parentheses is the offset in arcseconds between the IR position and the X-ray position in Table 3. 1. Source lies outside Spitzer coverage area. 2. Source is off-edge in IRAC 1-4. 3. Source is off-edge in IRAC 1 and 3. 4. Source is off-edge in IRAC 2 and 4. 5. Source appears extended in IRAC images. 6. IR position is offset by $>2$ " from X-ray position. Match is questionable. 7. Multiple IR sources near X-ray position. Possible source confusion. 8. HH 200-IRS. Previously identified in Table 1 of Devine et al. (2009) and as source 5 in Table 4 of Chapman \& Mundy (2009). The radio counterpart is L1228 VLA 4 in Table 2 of Reipurth et al. (2004). Source is extended in 2MASS, IRAC, and WISE images. Close IR companion (Figure 6). 9. WISE emission is likely variable (varflg $\geqslant 6$ in one or more bands). 10. Corresponds to source 9 in Table 4 of Chapman \& Mundy (2009) and source 11 of Kirk et al. (2009). 11. Optical counterpart is source 38 (OSHA 38) of Ogura \& Sato (1990). 12. Optical counterpart is source OSHA 42 in Table 1 of Kun et al. (2009) and source 42 of Ogura \& Sato (1990). 
Table 5

L1228 IR Photometry ${ }^{\mathrm{a}}$

\begin{tabular}{|c|c|c|c|c|c|c|c|c|c|c|c|c|}
\hline CXO Nr. & $J$ & $H$ & $K_{s}$ & $I 1$ & $I 2$ & $I 3$ & $I 4$ & $M 1$ & $W 1$ & $W 2$ & W3 & $W 4$ \\
\hline 1 & & & & $\ldots$ & 17.580 .27 & $\ldots$ & & $\ldots$ & & & & \\
\hline 2 & & & & $\ldots$ & 17.310 .23 & $\ldots$ & 16.160 .27 & $\ldots$ & & & & \\
\hline 3 & & & & $\ldots$ & $\ldots$ & $\ldots$ & $\ldots$ & $\ldots$ & & & & \\
\hline 4 & & & & $\ldots$ & $\ldots$ & $\ldots$ & $\ldots$ & $\ldots$ & & & & \\
\hline 5 & & & & $\ldots$ & $\ldots$ & $\ldots$ & $\ldots$ & 9.900 .19 & & & & \\
\hline 6 & & & & $\ldots$ & $\ldots$ & $\ldots$ & $\ldots$ & $\ldots$ & 17.730 .18 & 16.510 .22 & $13.27 \ldots$ & $9.57 \ldots$ \\
\hline 7 & & & & 15.980 .06 & 15.730 .12 & 15.170 .09 & 14.400 .11 & 10.890 .13 & 16.510 .07 & 15.880 .12 & $13.12 \ldots$ & $9.56 \ldots$ \\
\hline 8 & & & & $\ldots$ & $\ldots$ & $\ldots$ & $\ldots$ & . & 16.310 .06 & 15.420 .08 & 13.19 & 9.35 . \\
\hline 9 & & & & & & & & 9.130 .15 & 15.570 .04 & 14.910 .06 & 12.240 .23 & 9.120 .35 \\
\hline 10 & 13.880 .03 & 13.260 .03 & 13.070 .05 & 12.780 .05 & 12.760 .06 & 12.700 .05 & 12.640 .05 & $\ldots$ & 12.850 .02 & 12.650 .02 & 12.270 .24 & $9.30 \ldots$ \\
\hline 11 & & & & $\ldots$ & 16.710 .18 & $\ldots$ & 15.220 .18 & $\ldots$ & & & & \\
\hline 12 & & & & $\ldots$ & $\ldots$ & $\ldots$ & $\ldots$ & & & & & \\
\hline 13 & & & & $\ldots$ & $\ldots$ & $\ldots$ & $\ldots$ & $>10.80$ & & & & \\
\hline 14 & & & $>13.93$ & 13.390 .06 & 13.280 .07 & 11.850 .06 & 9.900 .06 & & 13.120 .02 & 12.850 .02 & 8.800 .02 & 6.730 .06 \\
\hline 15 & & & & 16.440 .06 & ... & 15.670 .10 & & & & & & \\
\hline 16 & & & & 17.960 .28 & 17.030 .20 & 16.300 .36 & 16.560 .30 & $>10.99$ & & & & \\
\hline 17 & & & & 18.510 .07 & $\ldots$ & $\ldots$ & $\ldots$ & $>11.86$ & & & & \\
\hline 18 & 10.390 .03 & 9.980 .03 & 9.980 .03 & 9.840 .05 & 9.840 .05 & 9.820 .05 & 9.780 .05 & 9.770 .11 & 9.790 .02 & 9.840 .02 & 9.870 .03 & 9.27 . \\
\hline 19 & & & & 17.880 .06 & $\ldots$ & 16.370 .21 & & & & & & \\
\hline 20 & & & & 17.450 .22 & 17.030 .20 & $\ldots$ & 15.950 .21 & & & & & \\
\hline 21 & & & & 17.590 .06 & 17.260 .23 & 17.040 .28 & 16.610 .33 & $\ldots$ & 17.540 .15 & $17.13 \ldots$ & $13.25 \ldots$ & $9.65 \ldots$ \\
\hline 22 & & & & $>17.85$ & & & & $\ldots$ & & & & \\
\hline 23 & & & & 15.090 .05 & 14.010 .07 & 12.920 .06 & 11.770 .06 & 8.480 .11 & 15.710 .04 & 14.000 .04 & 10.910 .07 & 8.580 .22 \\
\hline 24 & & & & 17.170 .05 & 16.710 .18 & 16.210 .06 & 15.880 .24 & & 16.740 .08 & 16.070 .14 & 12.490 .24 & 9.620 .45 \\
\hline 25 & & & & 16.710 .06 & 16.200 .05 & 16.030 .15 & 14.760 .06 & 10.920 .13 & & & & \\
\hline $26^{b, c}$ & 14.760 .09 & 13.560 .08 & 12.710 .05 & 11.370 .06 & 10.570 .06 & 9.760 .06 & 8.850 .05 & 3.380 .11 & 11.750 .02 & 10.630 .02 & 7.420 .01 & 3.430 .01 \\
\hline $27^{\mathrm{c}}$ & & & & 16.450 .13 & 15.170 .10 & 13.870 .08 & 13.320 .08 & $\ldots$ & 16.840 .09 & 14.690 .05 & 12.260 .20 & 7.580 .08 \\
\hline 28 & & & & 18.160 .06 & 17.300 .06 & 17.420 .13 & 17.180 .26 & & & & & \\
\hline 29 & 12.140 .02 & 11.480 .03 & 11.100 .02 & 10.660 .05 & 10.600 .06 & 10.510 .06 & 10.460 .05 & & 10.860 .02 & 10.560 .02 & 10.240 .04 & 8.740 .22 \\
\hline $30^{c}$ & 13.510 .03 & 11.980 .03 & 11.000 .02 & 9.960 .05 & 9.240 .05 & 8.760 .05 & 8.040 .05 & 4.910 .11 & 10.030 .02 & 9.140 .02 & 7.090 .01 & 4.720 .02 \\
\hline 31 & & & & 16.470 .05 & 16.040 .06 & 15.570 .06 & 15.260 .12 & & 16.910 .09 & 16.430 .19 & 12.150 .18 & 9.740 .53 \\
\hline 32 & & & & 16.210 .05 & 15.620 .05 & 14.920 .11 & 13.790 .06 & & & & & \\
\hline 33 & & & & 17.590 .06 & 16.650 .07 & 16.200 .07 & $>15.39$ & & & & & \\
\hline 34 & & & & 16.650 .05 & 16.330 .06 & 16.000 .06 & 14.710 .16 & & 17.120 .11 & 16.560 .21 & 12.260 .20 & $9.11 \ldots$ \\
\hline 35 & & & & 16.770 .05 & 15.740 .12 & 14.410 .05 & 13.610 .09 & 9.830 .11 & 17.730 .18 & 15.780 .11 & 11.400 .09 & 8.920 .25 \\
\hline 36 & 11.980 .02 & 11.050 .03 & 10.730 .02 & 10.600 .05 & 10.540 .06 & 10.470 .06 & 10.460 .06 & 10.600 .11 & 10.620 .02 & 10.490 .02 & 10.340 .04 & 8.910 .25 \\
\hline 37 & & & & 16.650 .05 & 16.060 .05 & 15.560 .06 & 14.890 .07 & 10.870 .11 & 16.680 .08 & 16.370 .18 & 12.440 .22 & 9.47. \\
\hline 38 & & & & 16.380 .05 & 15.650 .12 & 14.640 .06 & 13.720 .10 & 10.500 .12 & 16.740 .08 & 15.540 .10 & 12.040 .16 & 9.430 .41 \\
\hline 39 & & & & 17.360 .06 & 16.420 .06 & 15.750 .06 & 14.210 .06 & $\ldots$ & & & & \\
\hline 40 & & & & 18.200 .06 & 17.540 .06 & 16.560 .08 & 16.000 .09 & 12.030 .18 & & & & \\
\hline 41 & & & & 17.380 .05 & 16.590 .06 & 16.230 .19 & 15.240 .07 & 11.520 .14 & & & & \\
\hline 42 & & & & 16.780 .15 & 16.250 .16 & 15.860 .16 & 14.380 .12 & 10.400 .11 & & & & \\
\hline 43 & & & & 17.020 .05 & 16.450 .06 & 16.080 .06 & 15.830 .22 & $>10.77$ & & & & \\
\hline 44 & & & & 16.990 .05 & 16.190 .05 & 15.470 .06 & 14.260 .06 & 10.540 .11 & 17.090 .10 & 15.700 .10 & 11.700 .12 & 9.35 . \\
\hline 45 & 10.790 .02 & 10.500 .03 & 10.470 .02 & $\ldots$ & 10.480 .06 & & 10.430 .06 & & 10.410 .02 & 10.430 .02 & 10.370 .04 & $9.40 .$. \\
\hline 46 & & & & 16.390 .13 & 16.010 .13 & 15.760 .15 & 15.680 .20 & & & & & \\
\hline 47 & & & & 16.750 .05 & 15.780 .12 & 14.890 .11 & 13.880 .09 & 10.720 .12 & 16.900 .09 & 16.010 .13 & 12.000 .16 & 9.530 .45 \\
\hline 48 & & & & 16.340 .05 & 16.070 .06 & 15.710 .06 & 14.880 .18 & & 16.710 .08 & 17.160 .35 & 12.570 .25 & $9.68 \ldots$ \\
\hline 49 & 13.710 .03 & 13.130 .04 & 12.840 .03 & 12.600 .05 & 12.520 .05 & 12.420 .05 & 12.430 .05 & 11.650 .14 & 12.690 .03 & 12.530 .02 & 11.580 .10 & $9.16 \ldots$ \\
\hline 50 & & & & 16.290 .05 & 15.400 .05 & 14.520 .06 & 13.440 .06 & & 16.580 .07 & 15.120 .07 & 11.320 .09 & 8.990 .28 \\
\hline 51 & & & & 18.240 .06 & $\ldots$ & 16.950 .08 & .. & $>12.45$ & & & & \\
\hline 52 & & & & $\ldots$ & $\ldots$ & $\ldots$ & .. & $>13.87$ & & & & \\
\hline $53^{\mathrm{c}}$ & 11.510 .02 & 10.360 .03 & 9.700 .02 & $\ldots$ & $\ldots$ & $\ldots$ & $\ldots$ & 4.520 .11 & 8.750 .02 & 8.090 .02 & 6.500 .01 & 4.450 .02 \\
\hline 54 & & & & $\ldots$ & $\ldots$ & $\ldots$ & & $\ldots$ & & & & \\
\hline 55 & & & & 17.650 .06 & 16.960 .09 & 16.400 .08 & 15.060 .23 & $\ldots$ & & & & \\
\hline 56 & 11.900 .02 & 10.950 .03 & 10.550 .02 & 10.250 .05 & 9.920 .05 & 9.600 .05 & 8.840 .05 & 6.350 .11 & 10.290 .02 & 9.950 .02 & 7.950 .02 & 6.200 .04 \\
\hline 57 & & & & 18.210 .29 & $\ldots$ & $\ldots$ & $\ldots$ & & & & & \\
\hline 58 & & & & 15.830 .11 & 15.700 .12 & 15.200 .12 & 14.440 .14 & $\ldots$ & 15.840 .05 & 15.330 .08 & 11.870 .14 & 9.09 . \\
\hline 59 & & & & $\ldots$ & $\ldots$ & $\ldots$ & $\ldots$ & $\ldots$ & & & & \\
\hline 60 & 14.560 .04 & 13.990 .04 & 13.590 .05 & $\ldots$ & $\ldots$ & $\ldots$ & $\ldots$ & $\ldots$ & 13.490 .02 & 13.320 .03 & 11.430 .09 & 9.720 .53 \\
\hline $199^{\mathrm{d}}$ & 13.020 .03 & 10.610 .03 & 9.170 .03 & $\ldots$ & $\ldots$ & $\ldots$ & $\ldots$ & $\ldots$ & 7.890 .02 & 6.550 .02 & 3.880 .01 & 1.380 .01 \\
\hline
\end{tabular}

Notes.

a $J, H, K_{s}=2$ MASS. $I 1-I 4=$ IRAC bands $1-4$. $M 1=$ MIPS-24. $W 1-W 4=W I S E$ bands $1-4$. Each column gives source magnitude followed by its uncertainty.

$\mathrm{b}$ There is disagreement between 2MASS $J H K_{s}$ photometry and that obtained with the KPNO 4 m by Chapman \& Mundy (2009).

${ }^{c}$ IR emission may be variable. WISE varflg $\geqslant 6$ in one or more bands.

${ }^{\mathrm{d}} \mathrm{HH}$ 199-IRS. Not detected by Chandra. 


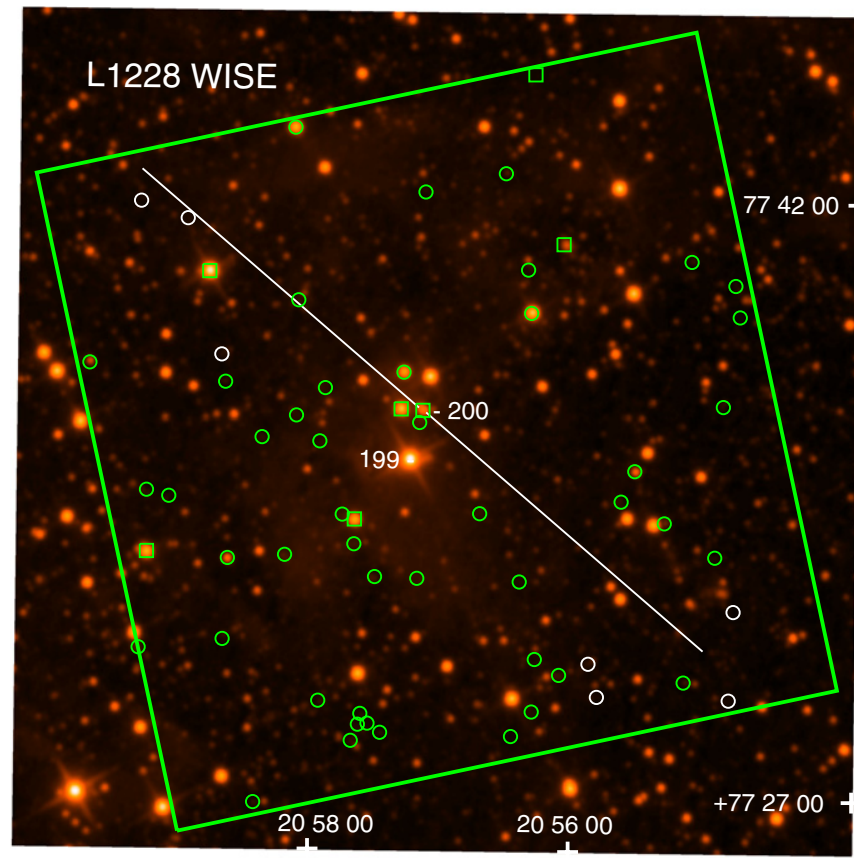

Figure 2. Positions of L1228 X-ray sources overlaid on a WISE W1 $(3.4 \mu \mathrm{m})$ image. Squares denote known or suspected YSOs. Objects marked in green have IR counterparts but only the brightest IR sources are visible in this image. Objects marked in white circles lack IR counterparts. The HH-driving sources HH 199-IRS (IRAS 20582+7724) and HH 200-IRS are marked. The solid lines show the HH 200 outflow axes at P.A. $=49^{\circ} / 229^{\circ}$. The emission knots associated with HH 199 are directed generally eastward (to the left) but span a range of position angles P.A. $=60^{\circ}-100^{\circ}$ (Devine et al. 2009; see also Figure 7). HH 199-IRS was not detected in X-rays. The Chandra ACIS-I detector footprint is shown as a $16.9 \times 16^{\prime} .9$ square. Log intensity scale. J2000 coordinates.

(A color version of this figure is available in the online journal.)

\subsection{X-Ray Variability}

$\mathrm{X}$-ray variability is a common feature of YSOs but only four L1228 sources (CXO 8, 10, 31, and 50) show a high probability of variability $P_{\mathrm{var}} \geqslant 0.9$. They are all relatively X-ray bright with $>50$ net counts (Figure 3 ). This suggests that variability could be present in some fainter sources but went undetected because of low count rates. The X-ray light curves of the variable sources are shown in Figure 4. In all cases the variability is rather low-level with count rates changing by factors of $\sim 2-3$. No large-amplitude X-ray flares were detected.

\subsection{X-Ray Spectra}

The analysis of X-ray spectra can potentially provide useful information on the line-of-sight neutral hydrogen absorption column density $\left(N_{\mathrm{H}}\right)$ toward the source and characteristic plasma temperatures $(k T)$ for thermal sources, or photon power-law (PL) indices $(\Gamma)$ for nonthermal sources such as active galactic nuclei (AGNs). In our experience, at least $\sim 50$ counts are needed to justify rudimentary spectral analysis and we have thus extracted spectra for the 8 sources with $>50$ counts. We attempted to fit the spectra with an absorbed isothermal (1T) thermal plasma model (the apec model in XSPEC) and also with an absorbed PL model for those sources that were classified as galaxies or AGNs based on their IR properties (Section 4). In all but two cases the simple $1 \mathrm{~T}$ model gave statistically acceptable results with realistic plasma temperatures of $k T \approx 0.8-6 \mathrm{keV}$. The two exceptions (CXO 10 and 50) are discussed below. The spectral fit results are summarized in Table 6.

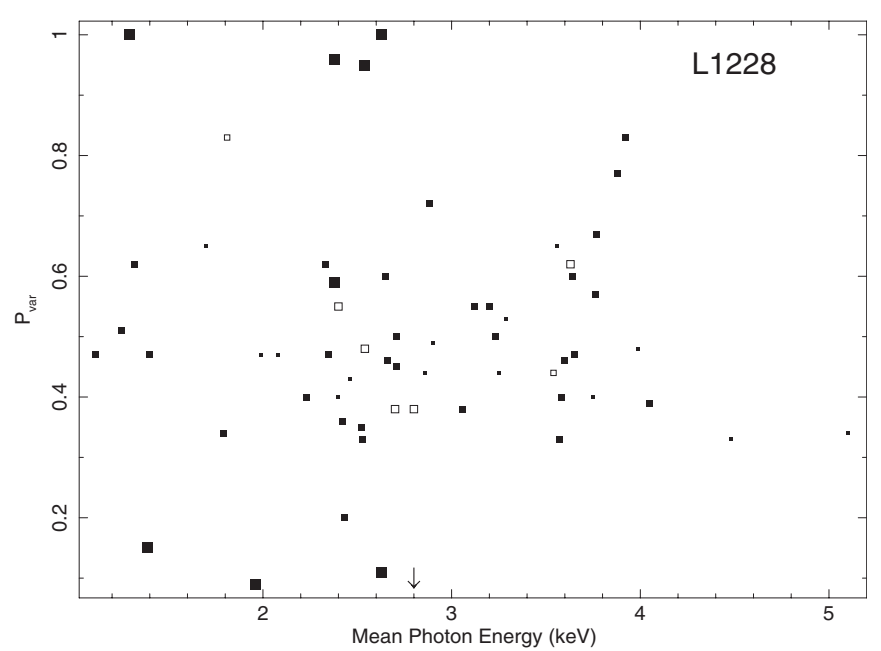

Figure 3. X-ray variability probability $P_{\text {var }}$ vs. mean photon energy for the X-ray sources detected in L1228. Symbol sizes reflect the number of X-ray counts: small $<10$ counts, medium $10-50$ counts, large $>50$ counts. Filled symbols denote sources with IR counterparts and open symbols lack counterparts. The arrow at $2.8 \mathrm{keV}$ marks the average value of the mean photon energy for the $\mathrm{X}$-ray sample. The source with the highest mean energy at far right is $\mathrm{HH} 200$ IRS. Those sources with $P_{\text {var }} \geqslant 0.9$ all have $>50$ counts (CXO sources 8,10 , 31 , and 50).

The median of the $N_{\mathrm{H}}$ values for the eight sources in Table 6 is $N_{\mathrm{H} \text {,med }}=5.25 \times 10^{21} \mathrm{~cm}^{-2}$, where we have used the average of the two slightly different $N_{\mathrm{H}}$ values for those sources fitted with both a thermal and a PL model. Using the conversion $N_{\mathrm{H}}=2.2 \times 10^{21} A_{\mathrm{V} \mathrm{cm}} \mathrm{cm}^{-2}$ of Gorenstein (1975), the median value is $A_{\mathrm{V} \text {,med }}=2.4 \mathrm{mag}$. The conversion $N_{\mathrm{H}}=1.6 \times$ $10^{21} A_{\mathrm{V}} \mathrm{cm}^{-2}$ of Vuong et al. (2003) gives $A_{\mathrm{V} \text {,med }}=3.3 \mathrm{mag}$. Although the sample is small, the latter value $A_{\mathrm{V} \text {,med }}=3.3$ based on the Vuong et al. conversion is nearly identical to the median $A_{\mathrm{V} \text {, med }}=3.1$ obtained in the optical study of Kun et al. (2009).

The 1T model did not give an acceptable spectral fit for CXO source 10 but a two-temperature (2T) model was acceptable. It is the brightest X-ray source in the sample and is variable. It is classified as a star in the Hubble Space Telescope (HST) Guide Star Catalog (GSC) (Table 3). Its X-ray variability is a sign of magnetic activity and if it is a YSO then it is likely a wTTS (Section 4.5). Multi-temperature thermal plasma models are often required to fit the spectra of X-ray bright TTSs where higher signal-to-noise ratio more tightly constrains the shape of the spectrum. Thus, the need for a $2 \mathrm{~T}$ model in the case of $\mathrm{CXO}$ 10 is not unusual and may simply reflect the higher quality of its spectrum.

CXO source 50 is also noteworthy. It is the second brightest $\mathrm{X}$-ray source and is classified as an AGN based on its Spitzer and WISE infrared properties (Section 4). But its X-ray emission is likely variable $\left(P_{\mathrm{var}}=0.98\right)$ and $\mathrm{X}$-ray variability is more commonly associated with magnetically active YSOs in starforming regions. Both $1 \mathrm{~T}$ and $2 \mathrm{~T}$ thermal models require a high plasma temperature component $k T \gtrsim 20 \mathrm{keV}$. Such a high temperature is well above the typical range $k T \sim 1-6 \mathrm{keV}$ for YSOs but is not impossible for a flaring magnetically active YSO. If the IR classification as an AGN is indeed correct, then the X-ray emission would more likely be nonthermal with a PL spectrum. We thus fitted the spectrum with an absorbed PL model and the fit is acceptable with a $\chi^{2}$ value nearly identical to that of the thermal model (Table 6). We thus cannot distinguish between a hot thermal plasma spectrum and a PL spectrum on the basis of fit statistics for CXO source 50 . 

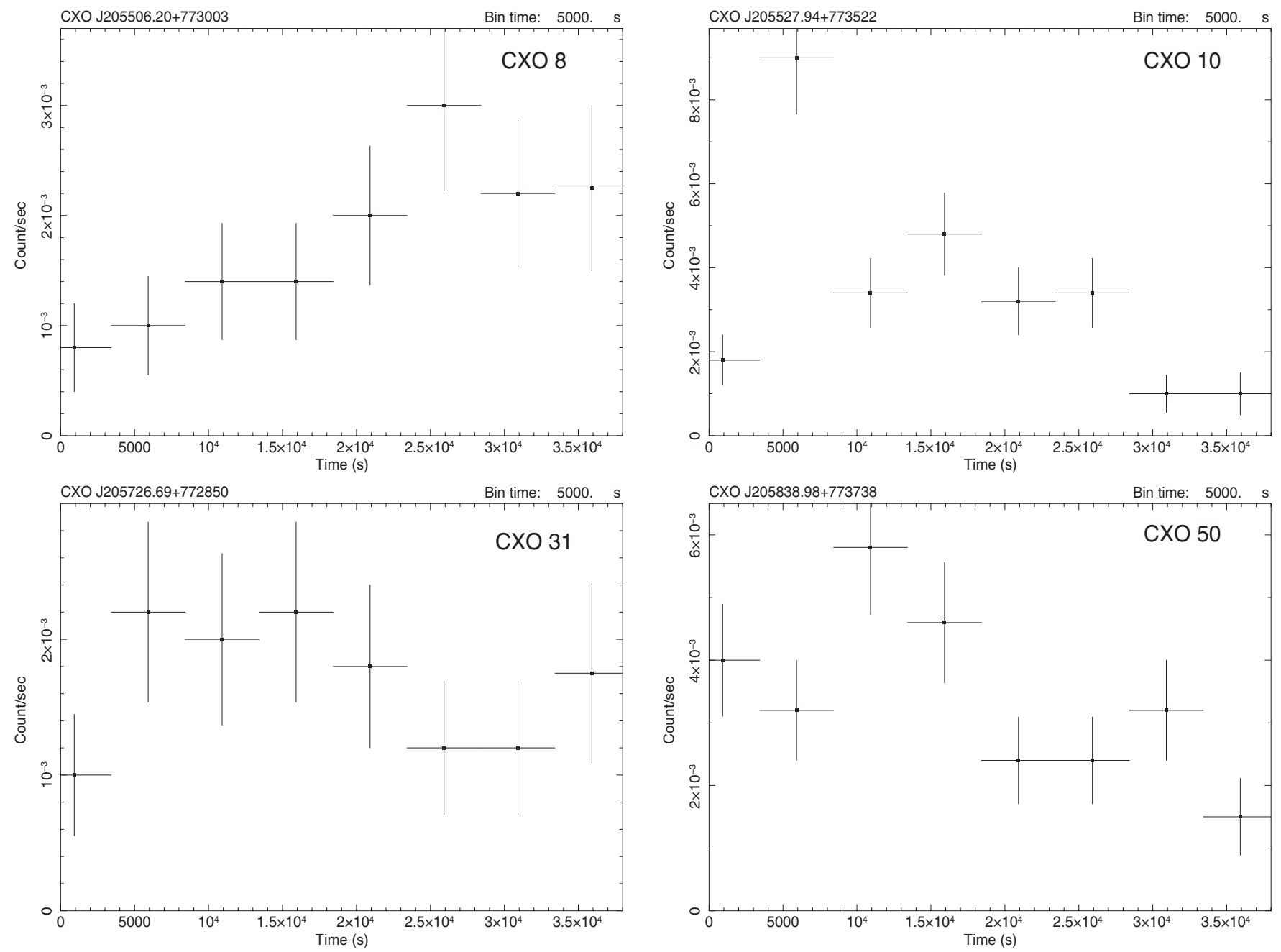

Figure 4. Chandra ACIS-I light curves $(0.3-7 \mathrm{keV})$ of L1228 X-ray sources with high probability of variability $P_{\mathrm{var}}>0.9$, binned at $5000 \mathrm{~s}$ intervals. (Supplemental data (FITS) of this figure are available in the online journal.)

\section{YSO IDENTIFICATION}

\subsection{Methods}

We have used multi-wavelength IR data to identify those $\mathrm{X}$-ray sources that are potential YSOs. Object classifications based on IR analysis are given in Table 3. We have used IR color cuts (where color refers to the magnitude difference in two different bands) and color-magnitude constraints to identify candidate YSOs in the presence of contaminants such as star-forming galaxies with enhanced polycyclic aromatic hydrocarbon (PAH) emission, AGNs, and shock emission knots. Several different color analysis methods have been discussed in the literature and we have adopted that of Gutermuth et al. (2008) for Spitzer data and Koenig et al. (2012) for WISE data. Similar IR analysis for the Taurus-Auriga region based on these methods was presented by Rebull et al. (2010, 2011). We have restricted our IR color analysis to those objects for which IR photometry is available in all four IRAC bands or all four WISE bands (or both). In our sample, 41 objects have four-band photometry from IRAC or WISE (or both), and 10 of these also have 2MASS $J H K_{s}$ photometry (Table 5).

The color space methods implement cuts based on the photometric properties of previously identified extragalactic sources, shock emission features, and YSOs. It should be emphasized that classification schemes based on IR color diagrams are statistical in nature and are not $100 \%$ reliable. Such schemes are tuned to finding IR-bright YSOs. Contamination from AGNs becomes significant for fainter IR sources and the contamination rate increases as the objects become fainter. The schemes of Gutermuth et al. (2008) and Koenig et al. (2012) thus also apply magnitude cuts to identify potential faint extragalactic sources. For example, the Gutermuth et al. criteria require [I2] > 13.5 mag along with other color constraints before an object is classified as a broad-line AGN. Even so, low-mass embedded YSOs can occupy these IR-faint regimes. Closely spaced sources can also affect color analysis results, especially for lower resolution MIPS and WISE data. We have inspected the IR images of all Chandra sources and those with more than one IR source within the Chandra position error ellipse are noted in Table 4.

IR color analysis is capable of distinguishing between heavily reddened class I protostars with infalling envelopes and class II objects (i.e., cTTS with accretion disks). Class III objects (wTTSs) have little or no infrared excess and are generally not identifiable on the basis of IR colors, as demonstrated below (e.g., CXO source 36). Once a YSO candidate has been identified, its status as a class I, II, or III object can also be discerned by fitting the IR spectral energy distribution (SED) to determine the IR spectral index $\alpha=d \log \left(\lambda F_{\lambda}\right) / d \log \lambda$ where $F_{\lambda}$ is the flux density at wavelength $\lambda$. In the approach of Haisch 
Table 6

Spectral Fits of Lynds 1228 Bright X-Ray Sources ${ }^{\mathrm{a}}$

\begin{tabular}{|c|c|c|c|c|c|c|c|}
\hline $\begin{array}{l}\mathrm{CXO} \\
\mathrm{Nr} .\end{array}$ & Model & $\begin{array}{c}N_{\mathrm{H}} \\
\left(10^{22} \mathrm{~cm}^{-2}\right)\end{array}$ & $\begin{array}{c}k T_{1} \\
(\mathrm{keV})\end{array}$ & $\Gamma$ & $\begin{array}{l}\text { Norm }_{1} \\
\left(10^{-5}\right)\end{array}$ & $\chi^{2} /$ dof & $\begin{array}{c}F_{x}(0.3-7 \mathrm{keV}) \\
\left(10^{-14} \mathrm{erg} \mathrm{cm}^{-2} \mathrm{~s}^{-1}\right)\end{array}$ \\
\hline 8 & $1 \mathrm{~T}$ & $1.10[0.25-3.04]$ & $2.20[0.83-9.88]$ & & 3.11 [1.52-11.9] & $7.39 / 8$ & $1.94(4.86)$ \\
\hline 8 & PL & $1.05[0.24-3.14]$ & $\ldots$ & $2.55[1.49-4.35]$ & $1.63[0.00-16.3]$ & $8.01 / 8$ & $1.92(7.59)$ \\
\hline 10 & $2 \mathrm{~T}^{\mathrm{b}}$ & $0.16[0.00-0.50]$ & $0.22[0.14-0.51]$ & $\ldots$ & $1.97[0.23-\cdots]$ & $7.61 / 7$ & $3.01(6.57)$ \\
\hline 29 & $1 \mathrm{~T}$ & $0.69[0.35-0.99]$ & $0.84[0.56-1.05]$ & $\ldots$ & $1.92[0.91-4.14]$ & $2.99 / 3$ & $1.20(6.17)$ \\
\hline 31 & $1 \mathrm{~T}$ & $0.70[0.06-1.78]$ & $4.09[1.54-\cdots]$ & $\ldots$ & $2.10[1.21-4.28]$ & $2.82 / 4$ & $2.13(3.56)$ \\
\hline 31 & PL & $0.66[0.04-1.71]$ & $\ldots$ & $1.95[1.02-3.12]$ & $0.77[0.00-3.03]$ & $2.92 / 4$ & $2.01(3.95)$ \\
\hline 38 & $1 \mathrm{~T}$ & $0.32[0.09-0.65]$ & $6.38[3.20-\cdots]$ & $\ldots$ & $3.26[2.55-4.31]$ & $11.2 / 11$ & $4.35(5.82)$ \\
\hline 38 & PL & $0.42[0.11-0.84]$ & $\ldots$ & $1.83[1.23-2.58]$ & $1.23[0.60-2.69]$ & $11.0 / 11$ & $4.06(6.69)$ \\
\hline $50^{\mathrm{e}}$ & $1 \mathrm{~T}$ & $0.31[0.12-0.69]$ & $20.4[4.35-\cdots]$ & $\ldots$ & $3.80[2.96-5.14]$ & $3.32 / 6$ & $4.96(6.25)$ \\
\hline $50^{\mathrm{e}}$ & PL & $0.35[0.07-0.75]$ & $\ldots$ & 1.44 [0.97-1.99] & $0.92[0.49-1.78]$ & $3.31 / 6$ & $4.84(6.47)$ \\
\hline 53 & $1 \mathrm{~T}$ & $0.20[0.01-1.45]$ & $2.71[0.49-4.79]$ & $\ldots$ & $1.73[1.21-12.4]$ & $6.62 / 8$ & $1.99(2.76)^{\mathrm{c}}$ \\
\hline 53 & $1 \mathrm{~T}$ & $\{0.65\}^{\mathrm{d}}$ & $1.60[1.22-2.19]$ & $\ldots$ & $2.22[1.63-2.85]$ & $8.58 / 9$ & $1.59(3.90)$ \\
\hline 55 & $1 \mathrm{~T}$ & $1.84[0.40-3.79]$ & $2.91[1.50-\ldots]$ & $\ldots$ & $4.25[1.78-10.8]$ & $4.04 / 4$ & $2.68(6.84)$ \\
\hline 55 & PL & $1.84[0.26-5.65]$ & $\ldots$ & $2.23[0.98-4.54]$ & $1.87[0.00-53.7]$ & $5.09 / 4$ & $2.56(8.81)$ \\
\hline
\end{tabular}

\section{Notes.}

a Based on XSPEC (ver. 12.7.1) fits of background-subtracted ACIS-I spectra binned to a minimum of 10 counts per bin, unless otherwise noted. The tabulated parameters are the CXO source number (Table 3); model type, where PL denotes a power-law model and 1T or 2T denotes the number of temperature components in the apec optically thin thermal plasma model; absorption column density $\left(N_{\mathrm{H}}\right)$; plasma energy $\left(k T_{1}\right)$; photon power-law index $(\Gamma)$ where $F_{x} \propto$ $E^{-(\Gamma-1)} ;$ XSPEC component normalization $\left(\right.$ norm $\left._{1}\right) ; \chi^{2}$ statistic/degrees-of-freedom; and the absorbed X-ray flux followed in parentheses by the unabsorbed flux. For those sources classified as galaxies or AGNs on the basis of IR colors, results of a PL model are given in addition to thermal models. Solar abundances were assumed for apec models and are referenced to the values of Anders \& Grevesse (1989). Square brackets enclose $90 \%$ confidence intervals and an ellipsis means that the algorithm used to compute confidence intervals did not converge.

${ }^{\mathrm{b}}$ A $1 \mathrm{~T}$ model fit is statistically unacceptable. The $2 \mathrm{~T}$ model requires an additional hot plasma component at $k T_{2}=1.43[1.22-1.95] \mathrm{keV}$ and norm $2=1.33$ [0.85-1.94]. The tabulated results are for the $2 \mathrm{~T}$ model.

${ }^{\mathrm{c}}$ Class II YSO (OSHA 42). At an assumed L1228 distance of $200 \mathrm{pc}$ the unabsorbed flux gives an X-ray luminosity log $L_{x}(0.3-7 \mathrm{keV})=29.12$ erg s ${ }^{-1}$.

${ }^{\mathrm{d}} N_{\mathrm{H}}$ held fixed at the value corresponding to $A_{\mathrm{V}}=3.42$ mag obtained by Kun et al. (2009). The unabsorbed flux gives $\log L_{x}(0.3-7 \mathrm{keV})=29.27$ erg s ${ }^{-1}$ at $d=200 \mathrm{pc}$.

e The spectrum was binned to a minimum of 15 counts per bin. A 1 T model requires a high but uncertain plasma temperature.

et al. (2001), $\alpha$ is evaluated in the $\approx 2-10 \mu \mathrm{m}$ range. Class I sources have $\alpha>0.3$, flat spectrum sources have $-0.3 \leqslant \alpha<$ 0.3 , class II sources have $-1.6 \leqslant \alpha<-0.3$, and class III sources have $\alpha<-1.6$. We have computed spectral indices for those sources suspected of being YSOs for comparison with classes assigned on the basis of IR colors.

\subsection{Extragalactic Contaminants}

Because of the modest extinction toward L1228 (median $A_{\mathrm{V}} \approx 3 \mathrm{mag}$ ) and its location above the galactic plane ( $b=$ $+20^{\circ}$ ), the X-ray sample is expected to contain a substantial number of extragalactic sources. Indeed, of the $41 \mathrm{X}$-ray sources with four-band IRAC or WISE mid-IR photometry suitable for color space analysis, 28 are classified as AGNs or starforming galaxies. This yields an estimated extragalactic source fraction of $28 / 41(68 \%)$. There are undoubtedly other as yet unidentified extragalactic sources in the sample since 12 sources with IR counterparts lacked sufficient photometry for colorbased classification and $7 \mathrm{X}$-ray sources have no counterparts. We also note here that nine X-ray sources having complete fourband IR photometry did not pass any of the color-based criteria for classification as AGN, PAH-emission star-forming galaxy, shock emission knot, or YSO. Optical catalog searches show that four of these are stars (CXO 10, 29, 36, and 45; see Table 3) but the other five objects remain unclassified (CXO 18, 28, 46, 49, and 60).

All eight of the brightest X-ray sources with $>50$ counts have four-band mid-IR photometry and five of them are classified as extragalactic on the basis of their IR properties (CXO 8, 31, 38,50 , and 55). We compare the above with the number of bright extragalactic X-ray sources ( $>50$ counts) predicted on the basis of source count data in the Chandra Deep Field-North (CDF-N) Survey as analyzed by Brandt et al. (2001). They show that the number of X-ray sources $N$ per square degree with an absorbed hard-band (2-8 keV) flux greater than a specified lower limit $F_{x \text {, hard }}$ is $N\left(>F_{x \text {, hard }}\right)=2820\left(F_{x \text {, hard }} /\right.$ $\left.10^{-15} \mathrm{erg} \mathrm{cm}^{-2} \mathrm{~s}^{-1}\right)^{-1.0 \pm 0.3} \mathrm{deg}^{-2}$. To apply this result, we need to know what value of $F_{x}$, hard is required to accumulate at least 50 counts $(0.3-7 \mathrm{keV})$ from a generic extragalactic source toward L1228 in a $36,779 \mathrm{~s}$ exposure. Note here that we require $>50$ counts in the broad $0.3-7 \mathrm{keV}$ band in order to make comparisons with the L1228 net counts measurements in Table 3. Following Brandt et al. we assume a PL X-ray spectrum with a photon PL index $\Gamma=1.4$ for the generic extragalactic source. We also assume a median absorption column density toward L1228 of $N_{\mathrm{H}}=5 \times 10^{21} \mathrm{~cm}^{-2}$ (Section 3.1). Under these spectral assumptions, the Portable Interactive Multi-Mission Simulator (PIMMS) ${ }^{8}$ simulator predicts an absorbed flux $F_{x \text {,hard }}=1.54 \times 10^{-14} \mathrm{erg} \mathrm{cm}^{-2} \mathrm{~s}^{-1}$. This value of $F_{x \text {,hard }}$ will yield 50 broad-band counts $(0.3-7 \mathrm{keV})$ and 27 hard-band counts $(2-8 \mathrm{keV})$ in $36,779 \mathrm{~s}$. Substituting this value of $F_{x \text {, hard }}$ into the above expression for source counts and scaling the result to the ACIS-I FoV $\left(0.07834 \mathrm{deg}^{2}\right)$ gives $N\left(>F_{x, \text { hard }}\right)=14(6-33)$ where the range in parentheses reflects the uncertainties in the CDF-N source count PL fit index. Thus, the hard-band CDF-N data predict at least 6 extragalactic sources in the L1228 FoV with $>50$ broad-band counts and we have identified 5 candidates. This small difference is not problematic since we have

\footnotetext{
8 For more information on the Portable Interactive Multi-Mission Simulator
} (PIMMS) see http://cxc.harvard.edu/ciao/ahelp/pimms.html. 

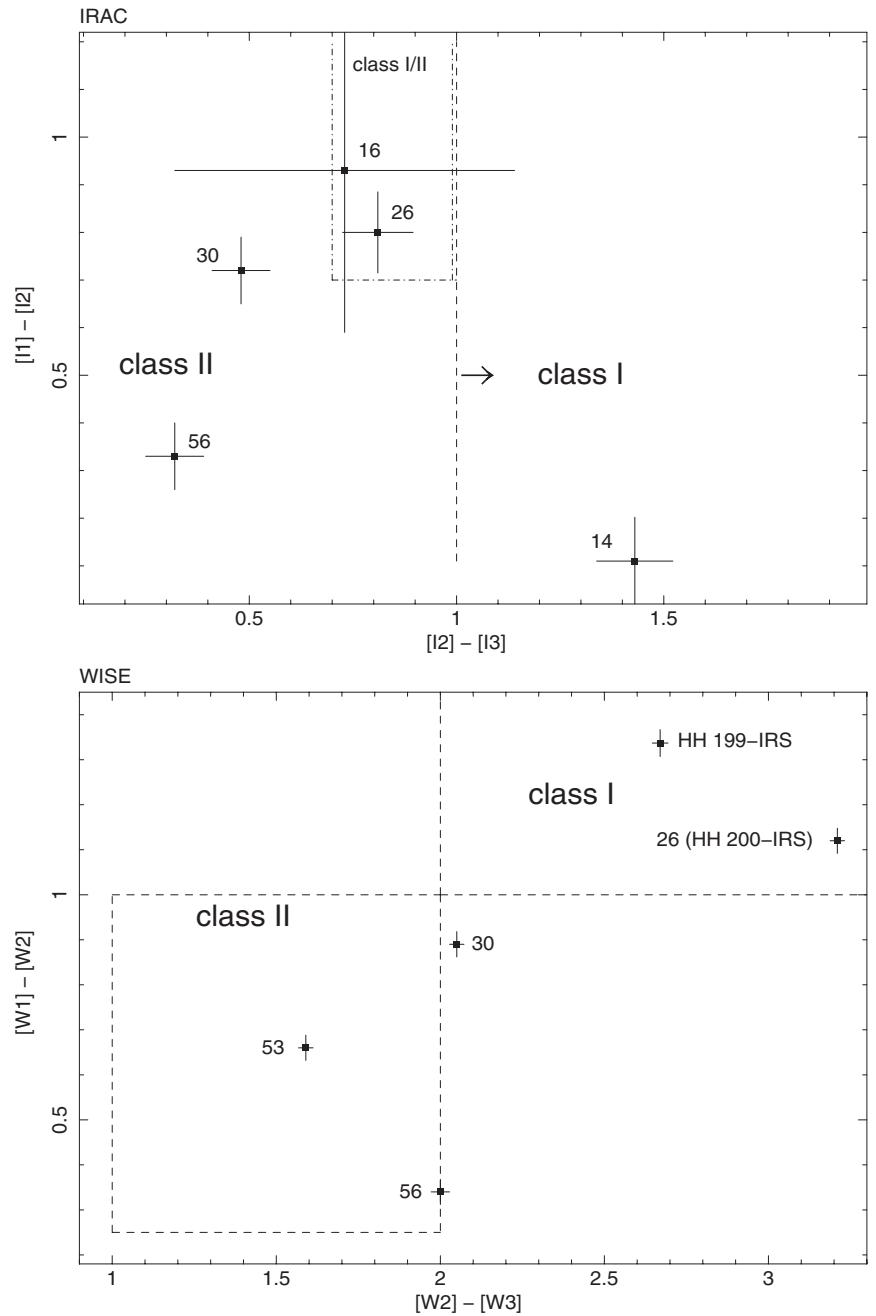

Figure 5. Representative color-color diagrams for Spitzer IRAC (top) and WISE (bottom) with colors of candidate YSOs shown. Source numbers correspond to Tables 3-5. The IRAC color-cut regions are from Gutermuth et al. (2008) and WISE regions are from Koenig et al. (2012). In the IRAC diagram, sources 16 and 26 lie in a region that includes both class I and heavily reddened class II sources. The WISE colors of HH 199-IRS are shown for comparison but it was not detected as an X-ray source.

assumed a constant $N_{\mathrm{H}}$ toward L1228 but the absorption is in fact higher toward the center of the cloud (Kun et al. 2009; Chapman \& Mundy 2009). In addition, some field-to-field variation in X-ray source counts is anticipated (Cowie et al. 2002).

\subsection{YSO Candidates}

We have identified seven YSO candidates in the X-ray sample (CXO sources 14, 16, 26, 30, 36, 53, and 56). All of these except CXO 36 were classified as YSOs on the basis of color analysis using four-band IRAC or WISE mid-IR photometry. Representative color-color diagrams for these objects are shown in Figure 5. CXO source 36 was unclassified on the basis of its IR colors but is designated a likely YSO by virtue of its association with $\mathrm{H} \alpha$ emission star number 38 (OSHA 38) in the list of Ogura \& Sato (1990). Our SED fits (Section 4.3) indicate that it has little if any IR excess so it is probably a class III object (wTTS).

Four of the YSOs are known from previous studies (CXO sources 26, 30, 36, and 53). CXO 26 is associated with the class I protostar HH 200-IRS (Chapman \& Mundy 2009). CXO 30 was identified as a class II YSO (cTTS) by Chapman \& Mundy (2009) and our analysis confirms this. CXO 36 is an $\mathrm{H} \alpha$ emission-line star as noted above. CXO 53 is associated with the emission-line star OSHA 42 (Kun et al. 2009), a class II object that has the strongest $\mathrm{H} \alpha$ emission of the stars identified in the survey of Ogura \& Sato (1990).

In addition to the above four previously known YSOs, CXO sources 14, 16, and 56 are classified as possible YSOs on the basis of IR color analysis. The YSO classifications of CXO 14 and 16 are questionable as discussed further below, but the classification of CXO 56 as a class II YSO on the basis of both Spitzer and WISE colors is relatively secure. The IR counterpart of CXO 56 is offset by only 0.48 from the X-ray position, making its association with the X-ray source highly probable.

Both CXO 14 and 16 are faint in X-rays and the latter object lies near the north edge of the ACIS-I detector (Figure 1) where sensitivity is degraded and the PSF is broadened. The Spitzer and WISE counterparts in Table 4 are offset from the X-ray position of CXO 14 by 3." 1 and 3."6 respectively. Similarly, the Spitzer counterpart of CXO 16 is offset by 3".6 from its $\mathrm{X}$-ray position. Even though these offsets are larger than for most of the other sources, the IR counterparts do lie within the Chandra $3 \sigma$ position error ellipses. The Spitzer source located 3". 1 SE of CXO 14 is resolved. This Spitzer source has IR colors consistent with a class I YSO but its corresponding WISE colors give a PAH-rich star-forming galaxy classification. Because of the above issues with mid-IR position offsets and IR color-class ambiguities, the classification of CXO sources 14 and 16 as YSOs will require further confirmation. Tighter constraints on their X-ray positions could be achieved by placing them more nearly on-axis where the Chandra PSF is sharpest and would provide a more reliable assessment of whether the Spitzer and WISE sources in Table 4 are legitimate counterparts.

\subsection{YSO Properties}

Four of the six objects identified as YSOs on the basis of IR colors have 2MASS near-IR photometry in addition to Spitzer or WISE photometry in at least four bands. Also, more recent near-IR photometry is available for CXO sources 26 and 30 from Chapman \& Mundy (2009). We have modeled the IR SEDs of these sources using the online modeling tool of Robitaille et al. (2007). The modeling results are summarized in Table 7. The fitting tool provides detailed information for several different models listed in order of increasing $\chi^{2}$ fit statistics. Substantial differences can be present for the value of any fitted parameter between different models. In Table 7 we give the median value of each parameter based on the five models with the lowest $\chi^{2}$ values. The most noteworthy result from the fits is the very high extinction toward the class I protostar HH 200-IRS (CXO 26). Such high absorption would absorb most soft X-ray photons and may thus be largely responsible for the low number of counts and high mean photon energy detected by Chandra.

We have also fitted the IR SED of CXO 36 since it is a $\mathrm{H} \alpha$ emission star, even though it was not classified as a YSO on the basis of its IR colors. The best-fit model gives no indication of a significant disk and the mass of any remnant disk must be very low $\left(M_{\text {disk }} \lesssim 10^{-8} M_{\odot}\right)$. A pure stellar photosphere Kurucz model with $T \sim 3750 \mathrm{~K}$ provides a good fit. The presence of $\mathrm{H} \alpha$ emission without a significant disk is indicative of a class III YSO (wTTS). This classification is further supported by its SED PL index $\alpha=-2.5 \pm 0.1$. 
Table 7

IR SED Model Fits of Lynds 1228 YSOs $^{\mathrm{a}}$

\begin{tabular}{|c|c|c|c|c|c|c|c|c|c|}
\hline $\begin{array}{l}\mathrm{CXO} \\
\mathrm{Nr} .\end{array}$ & $\begin{array}{c}A_{\mathrm{V}, \text { ism }} \\
\text { (mag) }\end{array}$ & $\begin{array}{l}A_{\mathrm{V}, \mathrm{cs}} \\
(\mathrm{mag})\end{array}$ & $\begin{array}{l}M_{\text {disk }} \\
\left(M_{\odot}\right)\end{array}$ & $\begin{array}{c}\dot{M}_{\mathrm{acc}} \\
\left(M_{\odot} \mathrm{yr}^{-1}\right)\end{array}$ & $\begin{array}{l}\text { Dist. } \\
\text { (pc) }\end{array}$ & $\begin{array}{l}L_{\text {tot }} \\
\left(L_{\odot}\right)\end{array}$ & $\alpha$ & $\begin{array}{l}\text { IR Class } \\
(\alpha)\end{array}$ & $\begin{array}{l}\text { IR Class } \\
\text { (colors) }\end{array}$ \\
\hline $14^{\mathrm{b}}$ & $\ldots$ & $\ldots$ & $\ldots$ & $\ldots$ & $\ldots$ & $\ldots$ & +1.38 & I & I \\
\hline $26^{c}$ & 5.0 & $>32^{\mathrm{c}}$ & $1.1 e-04$ & $2.0 e-06^{\mathrm{d}}$ & 191 & 0.66 & -0.04 & Flat & I \\
\hline 30 & 8.9 & $<0.35^{\mathrm{e}}$ & $1.3 e-03$ & $6.5 e-07^{\mathrm{d}}$ & 209 & 0.24 & -0.56 & II & II \\
\hline
\end{tabular}

a Based on fits of $J H K_{s}$ and Spitzer IRAC/MIPS-24 and WISE IR photometry using the Robitaille et al. (2007) modeling tool. The source number in the first column refers to Table 3. Tabulated parameters determined from the modeling tool are the interstellar and circumstellar extinctions, disk mass, mass accretion rates (sum of disk and envelope contributions), best-fit distance (constrained during fitting to lie within the range $180-220 \mathrm{pc}$ ), and total system luminosity $L_{\text {bol }}$. The quoted values are the median values of the five fitted models with the lowest $\chi^{2}$ fit statistic, unless otherwise noted. The IR spectral index $\alpha$ is based on power-law fits over the $\approx 2-8 \mu \mathrm{m}$ range where $\alpha=d \log \left(\lambda F_{\lambda}\right) / d \log \lambda$. The IR class is based on the spectral index criteria of Haisch et al. (2001) and that determined from mid-IR colors (see Figure 5).

b No SED modeling performed due to the lack of near-IR photometry.

${ }^{\mathrm{c}} \mathrm{HH} 200$-IRS. The fit used more recent $J H K_{s}$ photometry from Chapman \& Mundy (2009) instead of $2 \mathrm{MASS}$ data. The $A_{\mathrm{V}, \mathrm{cs}}$ limit is the lowest value obtained from the set of best-fit models.

${ }^{\mathrm{d}}$ Parameter is not tightly constrained by IR SED models. Fitted values for different models span an order of magnitude.

e The $A_{\mathrm{V}, \mathrm{cs}}$ limit is the largest value obtained from the set of best-fit models.

\subsection{Other Interesting Sources}

Bright variable $x$-ray sources ( $C X O$ 8, 10, 31, and 50). As already noted in Section 3.2, four of the brightest X-ray sources have a high probability of variability $P_{\mathrm{var}} \geqslant 0.9$. Three of these are classified as AGNs or galaxies on the basis of their IR colors ( $C X O$ sources 8, 31, and 50). No counterparts were found for these three sources in a search of several galactic and extragalactic databases including the HST GSC version 2.3.2, Hipparcos, and USNO B1 catalogs. Nevertheless, since X-ray variability is a common trait of YSOs in star-forming regions, deep follow-up observations and time-monitoring of these three sources would be worthwhile in order to confirm that they are indeed extragalactic in nature. The fourth variable X-ray source (CXO 10) is the brightest X-ray detection and was unclassified on the basis of IR colors but has an optical counterpart in the HST GSC (Table 3). Its IR spectral index is $\alpha=-2.45 \pm 0.1$ which would only be consistent with a class III object if it is a YSO.

$C X O$ 27. This faint hard X-ray source was classified as an AGN on the basis of both IRAC and WISE colors. It lies just 19" south of HH 200-IRS (Figure 7). Its IR emission may be variable in the WISE W1 band (varflg $=91 \mathrm{nn}$ ). Because of its possible IR variability and projected position close to other known YSOs, follow-up observations of CXO 27 would be useful to determine if it is a YSO masquerading as an AGN. If it is a YSO then its IRAC spectral index $\alpha=+1.0$ [+0.1-+1.9; $90 \%$ conf. range] would be consistent with either a class I or flat-spectrum source.

CXO 29. The bright X-ray source CXO 29 was unclassified by IR colors and it also has an optical counterpart in the HST GSC classified as a star (Table 3). Its IR spectral index $\alpha=$ $-2.24 \pm 0.13$ would imply a class III object if it is a YSO.

\section{HERBIG-HARO ENERGY SOURCES}

\subsection{HH 199-IRS}

HH 199-IRS (= IRAS 20582+7724 = 2MASS J205712.94 +773543.7 ) is the brightest infrared source in the L1228 cloud core and is believed to power the giant $\mathrm{HH} 199$ molecular outflow (Bally et al. 1995; Devine et al. 2009). This object was not detected by Chandra nor were any of the optical emission knots in its outflow identified by Devine et al. (2009). Nevertheless, because of its IR brightness and key role as an $\mathrm{HH}$ driving source we summarize its IR properties. The discussion below is restricted to 2MASS and WISE data since IRAC fluxes are affected by saturation.

Based on WISE colors (Figure 5) it is classified as a class I protostar by the Koenig et al. (2012) criteria. A fit of its SED using 2MASS and WISE photometry gives a spectral index $\alpha=$ $+0.32 \pm 0.32$ ( $90 \%$ confidence errors). This again gives class I using the criteria of Haisch et al. (2001) but the uncertainties are large enough to allow a flat-spectrum source classification (i.e., borderline case between class I and class II). No significant IR variability was found by WISE (varflg $=2214$ ) but it is worth noting that its outflow exhibits large changes in morphology over time and may be precessing (Bally et al. 1995; Devine et al. 2009).

We have modeled the IR SED of HH 199-IRS using 2MASS and WISE photometry (Table 5; Figure 8). If the interstellar extinction is loosely constrained to be $A_{\mathrm{V}}$,ism $<20 \mathrm{mag}$, then the Robitaille et al. tool finds a good fit with a total extinction $A_{\mathrm{V}}$ $=A_{\mathrm{V}, \mathrm{ism}}+A_{\mathrm{V}, \mathrm{cs}}=14.7$ [14.5-16.1] mag, almost all of which is attributed to the interstellar component. The total system luminosity is $L_{\text {bol }}=7.0$ [2.7-9.6] $L_{\odot}$ assuming a distance $d=200 \pm 20 \mathrm{pc}$. These are the median values of the five best-fitting models followed in brackets by the range of values. The above luminosity is at least an order of magnitude greater than that of HH 200-IRS if both objects are at similar distances. The inferred disk mass is $M_{\text {disk }} \approx 0.026$ [0.022-0.032] $M_{\odot}$ with a disk accretion rate $\dot{M}_{\text {disk }}=2.6[1.5-7.2] \times 10^{-7} M_{\odot} \mathrm{yr}^{-1}$. If we enforce a tighter constraint $A_{\mathrm{V} \text {,ism }}<13$ mag based on the maximum L1228 core extinction determined by Kirk et al. (2009), then the modeling tool compensates by adding in additional circumstellar absorption but the resulting fit has larger residuals than the one summarized above. Thus, in terms of goodness of fit, the models that invoke relatively high interstellar extinction with low circumstellar extinction are favored. 


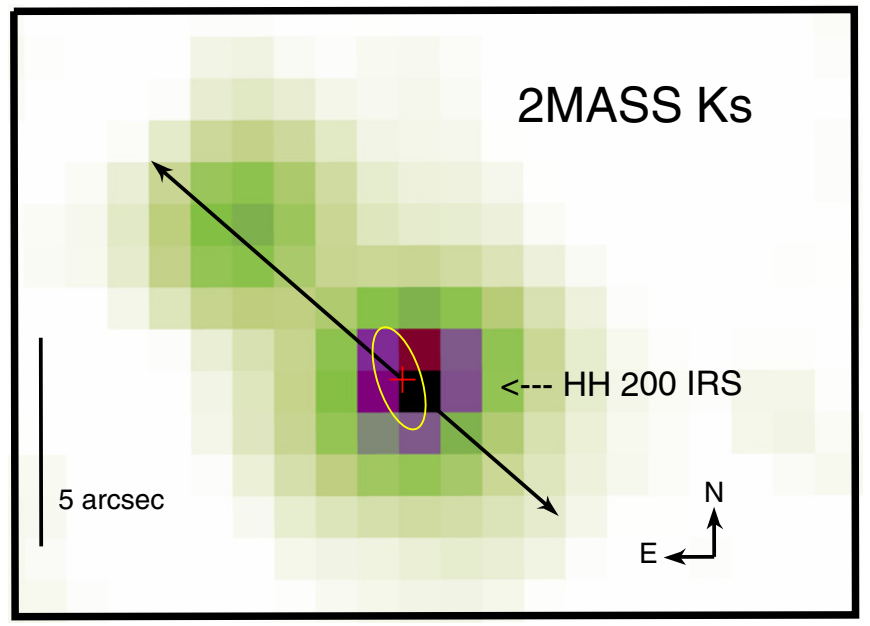

Figure 6. $2 \mathrm{MASS} K_{s}(2.159 \mu \mathrm{m})$ image of $\mathrm{HH} 200$-IRS $=2 \mathrm{MASS}$ J205706.55+773655.94. The cross marks the radio counterpart position of source VLA $4=\mathrm{J} 205706.703+773656.07$ (Reipurth et al. 2004). The X-ray position error ellipse for CXO source 26 (J205706.72+773656.1) is overlaid and has semi-axes 1".28 $\times 0$ 0.52. The ellipse is centered almost exactly on the VLA position. The arrows show the direction of the HH outflow axis at P.A. $=49^{\circ} / 229^{\circ}$ (Devine et al. 2009). The second fainter source located 5!'7 northeast of HH 200-IRS is 2MASS J205707.89+773659.7 $(J=15.76$, $H=14.24, K_{s}=13.44$ ) and was not detected by Chandra.

(A color version of this figure is available in the online journal.)

\subsection{HH 200-IRS}

This source shows a nearly flat IR SED in the 3-8 $\mu \mathrm{m}$ range but it then rises steeply at longer wavelengths (Figure 8). WISE colors are consistent with a class I source (Figure 5). Although its IR emission is bright it is only faintly detected in X-rays. The X-ray emission is hard with all detected photons having energies $E \geqslant 3.7 \mathrm{keV}$. The extinction toward this class I source is high. The five best-fit SED models using the Robitaille et al. tool (Table 7) yield a median interstellar extinction $A_{\mathrm{V}, \text { ism }}=$ 5 [3-13] mag but an additional large circumstellar extinction is also required. We examined several different models, all of which required $A_{\mathrm{V}, \mathrm{cs}}>32 \mathrm{mag}$. This implies that the central protostar is heavily obscured by a surrounding envelope. Any soft photons $(E<2 \mathrm{keV})$ emitted by the central source will thus be heavily absorbed and undetected. Because of the high absorption, the intrinsic X-ray properties of the central source are quite uncertain but the presence of sufficient hard emission to penetrate the dense circumstellar envelope is a strong clue that magnetic processes are involved in the X-ray production. Shocks created by impact of the outflow with surrounding material are expected to produce only soft emission $(E \lesssim 1 \mathrm{keV})$ which would be undetected unless viewed through lower absorption at large offsets from the star. We have checked for faint X-ray emission at the position of the optical emission knots in the $\mathrm{HH}$ 200-IRS outflow listed in Table 1 of Devine et al. (2009) and none was detected by Chandra.

A second IR source HH 200-IRSB (= 2MASS J205707.89 +773659.7 ) lies 5.77 northeast of HH 200-IRS. As Figure 6 shows, HH 200-IRSB is well outside the Chandra position error ellipse and is not detected in X-rays. Figure 7 is a Spitzer threecolor image of the region. This second source lies nearly on the HH 200 outflow axis, thus raising the question of whether it might actually be driving the giant $\mathrm{HH}$ outflow. This possibility cannot be totally ruled out but we believe that HH 200-IRS is more likely to be the driving source because of its elevated activity as evidenced by hard X-ray and radio emission (Reipurth et al. 2004).

Figure 8 compares the SEDs of HH 200-IRS and the close IR companion. The pair is resolved by 2MASS and IRAC but not by MIPS or WISE. Overall, the SEDs of the two sources look

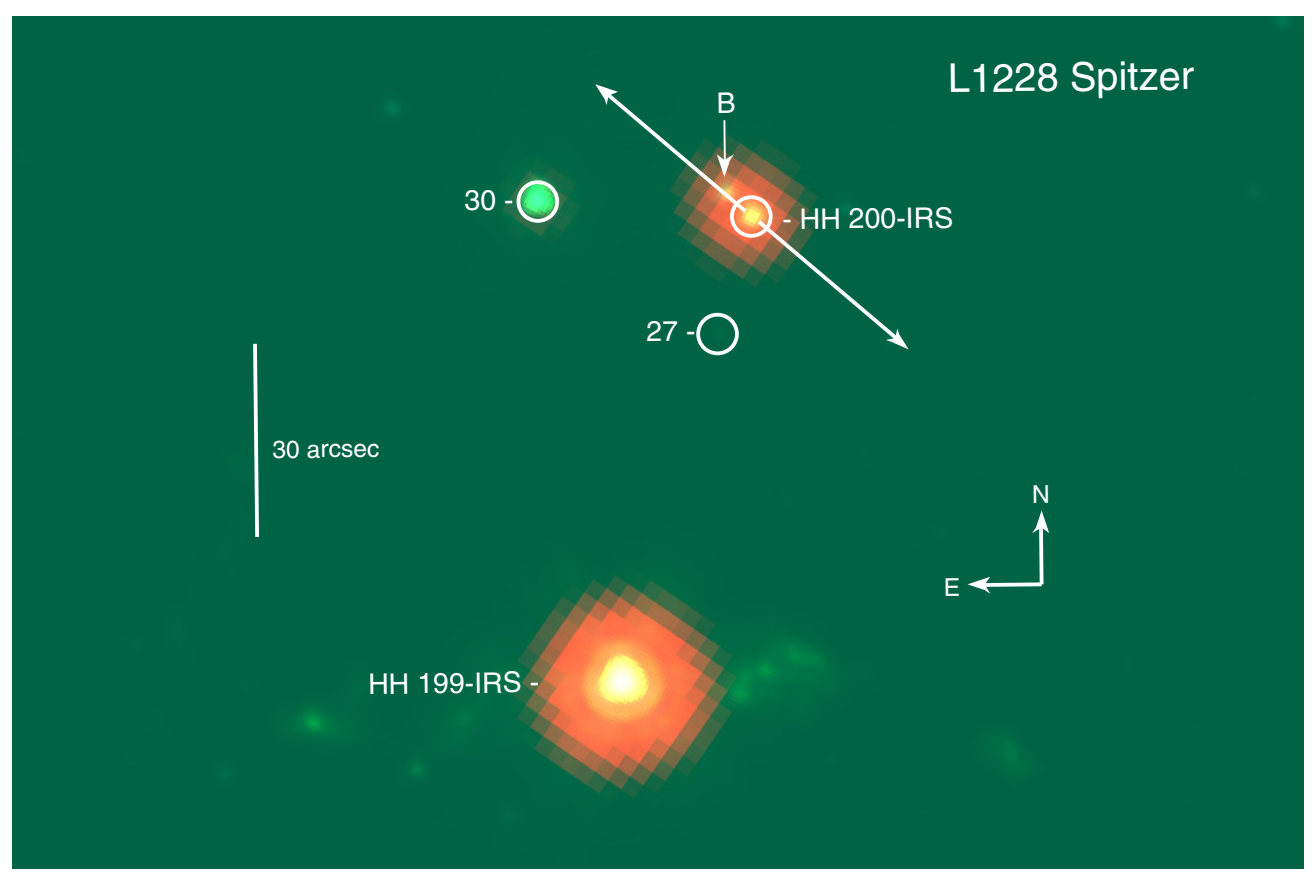

Figure 7. Gaussian-smoothed Spitzer red-green-blue (RGB) image of the region near HH 199-IRS and HH 200-IRS. $R=$ MIPS-24 $(24 \mu \mathrm{m}), G=I 2(4.5 \mu \mathrm{m}), B=I 1$ $(3.6 \mu \mathrm{m})$. The solid lines show the directions of the HH 200 bipolar outflow. Circles mark the positions of Chandra X-ray sources $(\mathrm{HH} 200-\mathrm{IRS}=\mathrm{CXO}$ nr. 26). The IR source marked B (= 2MASS J205707.89+773659.7) is located on the outflow axis 5'7 northeast of HH 200-IRS but was not detected by Chandra. HH 199-IRS was not detected by Chandra. Faint nebulosity associated with the HH 199 outflow is visible to the east and west of HH 199-IRS.

(A color version of this figure is available in the online journal.) 

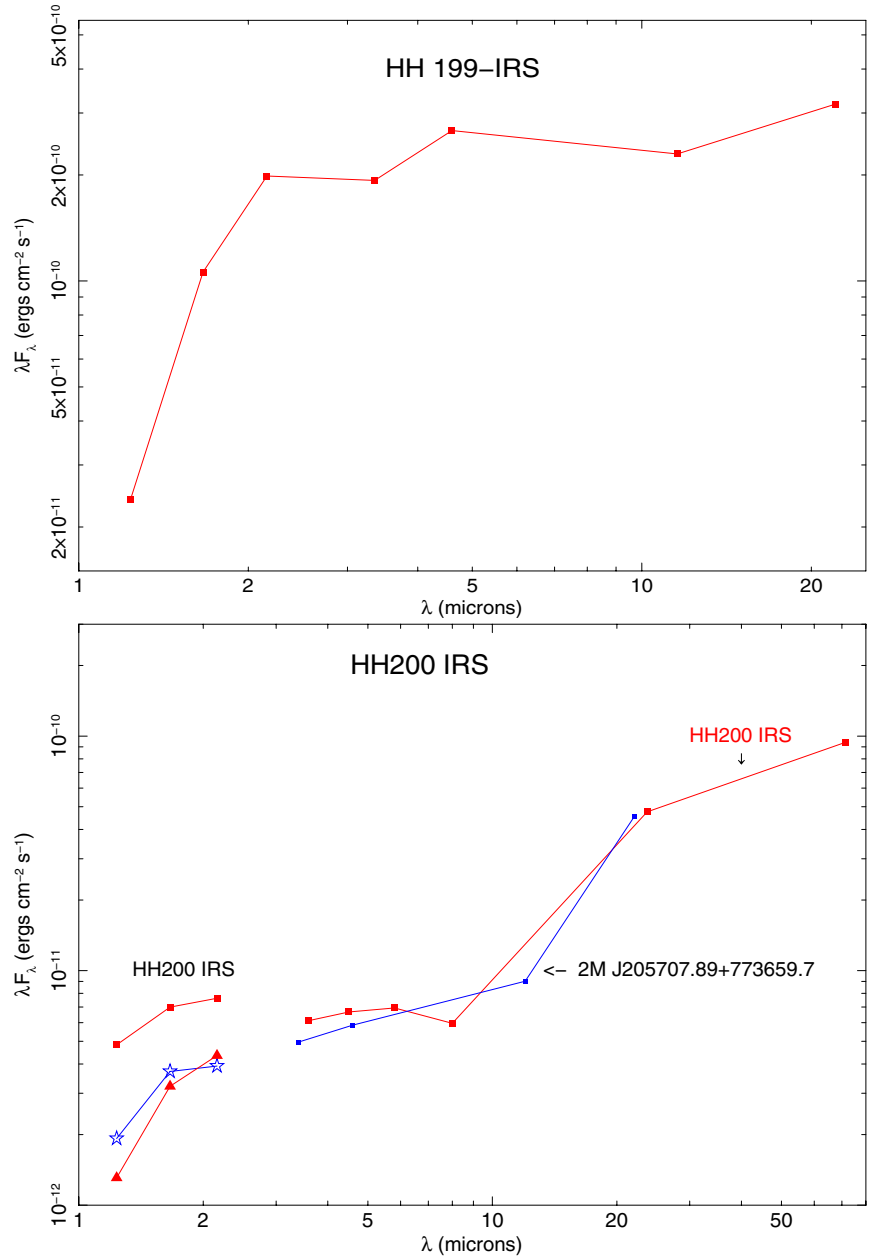

Figure 8. Top: IR SED of HH 199-IRS based on 2MASS and WISE photometry (Table 5). Bottom: IR SEDs of HH 200-IRS (red) and the close IR companion 2MASS J205707.89+773659.7 (blue) located 5".7 northeast of HH 200-IRS. The $J H K_{s}$ data for HH 200-IRS are from 2MASS (squares) and Chapman \& Mundy (2009; triangles). The MIPS-70 $\mu \mathrm{m}$ data point is from c2d. The two sources are only partially resolved by MIPS at $24 \mu \mathrm{m}$ and are unresolved by MIPS at $70 \mu \mathrm{m}$. The pair is not fully resolved by WISE.

(A color version of this figure is available in the online journal.)

quite similar and both are likely in the protostellar stage. As Figure 8 shows, there is a discrepancy between the $J H K_{s}$ fluxes of HH 200-IRS between 2MASS and the more recent values obtained by Chapman \& Mundy (2009) using the KPNO $4 \mathrm{~m}$. The $4 \mathrm{~m}$ fluxes are a factor of $\sim 4$ lower than 2MASS at $J$ band and a factor of $\sim 2$ lower at $H$ and $K_{s}$. This difference may be largely due to the better spatial resolution of the KPNO $4 \mathrm{~m}$ but some IR variability may also be present. The WISE variability flag has values varflg $=6631$ where a value of 6 in both $W 1$ and $W 2$ bands means that the source is potentially variable with small amplitudes.

\subsection{HH 199-IRS and HH 200-IRS in Comparison}

Our SED models indicate that $L_{\text {bol }}$ for $\mathrm{HH}$ 199-IRS is about 10 times greater than $\mathrm{HH}$ 200-IRS, in agreement with other studies (Chapman \& Mundy 2009; Kirk et al. 2009). The extinction toward the two protostars determined from SED fits is high and models require substantial circumstellar absorption in the case of HH 200-IRS. Faint X-ray emission was detected from HH 200-IRS but HH 199-IRS was undetected. Differences in the column density of X-ray absorbing gas toward the two protostars or their intrinsic X-ray properties could account for this. The high median photon energy of the events detected from $\mathrm{HH}$ 200-IRS is a clear sign that its intrinsic X-ray spectrum contains a hard component, undoubtedly of magnetic origin. $\mathrm{X}$-ray flares in class I protostars can produce such hard emission and in some cases the protostar only becomes detectable during an X-ray flare (Imanishi et al. 2001). If HH 200-IRS was in an active flare state during our Chandra observation then that could explain why it was detected as a hard source but HH 199-IRS was not. Variability is expected during an X-ray flare but we lack sufficient counts in HH 200-IRS to obtain a definitive test for $\mathrm{X}$-ray variability, but IR variability is suspected as mentioned above.

\section{THE NATURE OF THE L1228 CORE X-RAY POPULATION}

The Chandra observation reported here comprises the first deep X-ray census of the L1228 cloud core. The area observed by Chandra covers a relatively small $0.28 \times 0.28 \mathrm{FoV}$ and thus does not capture the entire L1228 cloud for which the region of highest extinction spans roughly $1 \mathrm{deg}^{2}$ (Figure 9 of Kun et al. 2009).

A large fraction of the $60 \mathrm{X}$-ray sources appears to be extragalactic which is not surprising given that L1228 lies well above the Galactic plane $(b=+20.2)$ and the cloud opacity is low (Lynds 1962). We have identified seven known or suspected YSOs. This $12 \%$ YSO fraction should be interpreted as a lower limit. We lack sufficient information for 19 X-ray sources to assess their YSO status, either because no optical or IR counterpart was found or because IR/optical data were insufficient to assign an object class.

It is of some interest that very few class III sources (wTTS) have so far been identified in L1228, but some have been found in other dark clouds in the Cepheus Flare and in off-cloud regions (Tachihara et al. 2005; Kirk et al. 2009). Since wTTS have weak $\mathrm{H} \alpha$ emission by definition and lack significant IR excesses they can be overlooked in $\mathrm{H} \alpha$ and infrared surveys. Of the 23 YSO candidates in L1228 identified in the IR study of Kirk et al. (2009), only 1 is classified as a wTTS. That object (Kirk et al.; nr. 136) lies outside the Chandra FoV but was not reported as an RASS X-ray detection by Tachihara et al. (2005). Is the wTTS population in L1228 truly absent or simply not yet seen? Some of the X-ray sources in our sample that lack IR color classifications may be wTTS, two of which have already been mentioned (CXO 10 and 29). Follow-up observations to search for stellar counterparts and spectral signatures of youth such as Li will be needed to determine whether any of the unclassified X-ray sources are indeed wTTS.

Another point worth noting is that the X-ray luminosities of the L1228 YSO candidates are quite low. The seven YSO candidates have a median of 12 net counts with a range spanning 5-75 net counts. Assuming a generic TTS thermal X-ray spectrum with $k T \sim 3 \mathrm{keV}$ and $A_{\mathrm{V}} \approx 3$ mag (Kun et al. 2009), this corresponds to unabsorbed luminosity $\log L_{x}(0.3-7 \mathrm{keV})=28.43$ [28.05-29.23] $\mathrm{erg} \mathrm{s}^{-1}$ at the nominal L1228 distance of $200 \mathrm{pc}$. These values increase by 0.35 dex at the high end of the distance range $(d=300$ pc; Kun 1998 ) and by about 0.17 dex for the higher mean extinction $A_{\mathrm{V}}$ $\approx 7$ mag obtained by Kirk et al. (2009). Based on the known correlations between $L_{x}$ and $M_{*}$ for TTS in Orion and Taurus, the above $L_{x}$ range corresponds to lower mass TTS with $M_{*} \lesssim$ $0.7 M_{\odot}$. (Figure 3 of Preibisch et al. 2005; Figure 1 of Telleschi et al. 2007). Thus, if a similar $L_{x} \propto M_{*}$ correlation holds for 
L1228, we are detecting a highly dispersed low-mass population of PMS stars that apparently is deficient in the more X-ray luminous TTS with masses $M_{*} \gtrsim 1 M_{\odot}$. This conclusion would also apply to any of the unclassified X-ray sources that may turn out to be YSOs. They have 6-37 net counts or $\log L_{x}=$ $28.13-28.92 \mathrm{erg} \mathrm{s}^{-1}$ (at $d=200 \mathrm{pc}, A_{\mathrm{V}}=3 \mathrm{mag}, k T=3 \mathrm{keV}$ ).

Some additional support for the dearth of higher mass TTS comes from the optical study of L1228 by Kun et al. (2009). Of the 13 PMS stars they identified in L1228, only one was more massive than $1 M_{\odot}$ and the median mass was $0.5 M_{\odot}$. One of the stars in their sample, the class II source OSHA 42, was the brightest X-ray source classified as a YSO in our sample. Kun et al. classify OSHA 42 as spectral type M0 with an estimated mass $M_{*}=0.6 M_{\odot}$ and $L_{\text {bol }}=0.49 L_{\odot}$. The value we obtain for $L_{\mathrm{bol}}$ from IR SED modeling is nearly identical (Table 7). To the extent that $L_{x}$ correlates with $M_{*}$ in L1228, it thus seems likely that the remaining L1228 X-ray sources identified as TTS (class II or possible III) with fainter emission than OSHA 42 are lower mass objects.

\section{SUMMARY}

The main results of our combined X-ray/infrared study of L1228 are summarized below.

1. Chandra has provided the first sensitive pointed X-ray observation of the Lynds 1228 cloud core. Previous optical, IR, and radio studies have shown that L1228 contains a modest population of low-mass PMS stars and the heavily reddened protostars HH 199-IRS and HH 200-IRS. Chandra detected $60 \mathrm{X}$-ray sources, most of which are faint ( $<40$ counts) and non-variable.

2. Using reprocessed Spitzer archive data, along with $2 \mathrm{MASS}$ near-IR data and recent WISE All-Sky Survey mid-IR data, IR counterparts were found for 53 sources. Source classification based on IR colors and magnitudes was undertaken on 41 of these for which photometry in at least four IRAC or WISE bands was available.

3. Seven Chandra sources are classified as YSOs, four of which were already known from previous studies. In addition, three X-ray sources have optical counterparts in the HST GSC or Tycho catalogs that are classified as stars but it remains to be determined whether they are PMS stars. We have classified 28 of the X-ray sources as extragalactic on the basis of their IR properties but additional extragalactic contaminants are likely present among the 19 X-ray sources that lack sufficient IR data to assign object classes. But some of the unclassified X-ray sources may be wTTSs, which are conspicuously absent in previous YSO surveys of L1228. Evidence that some wTTS may be present comes from the X-ray detection of $\mathrm{H} \alpha$ emission-line star OSHA 38 whose IR SED shows no evidence for a significant disk.

4. Disk properties were inferred for four of the YSOs having good-quality IR photometry using the SED modeling tool of Robitaille et al. (2007). Similar SED modeling was undertaken for the protostars HH 199-IRS and HH 200IRS.

5. Faint hard X-ray emission was detected from HH 200-IRS, the suspected driving source of the HH 200 giant outflow. Its IR colors confirm that it is a class I protostar and there are hints that its IR emission is variable. A close IR companion located 5".7 away also has a rising IR SED characteristic of a protostar but was undetected by Chandra. A very high circumstellar extinction is inferred for HH 200-IRS from
SED modeling, which likely accounts for its hard X-ray emission. No X-ray emission was detected from HH 199IRS but its IR colors and spectral index yield a class I protostar classification. Modeling of its IR SED shows that it is at least an order of magnitude more luminous than $\mathrm{HH}$ 200-IRS if it lies at the same distance.

6. The X-ray luminosities of the TTSs in L1228 detected by Chandra are low: $\log L_{x} \lesssim 29.7 \mathrm{erg} \mathrm{s}^{-1}$, assuming $A_{\mathrm{V}}$ and distances at the high end of the L1228 range. If a correlation between $L_{x}$ and stellar mass exists among TTS in L1228 similar to that in Taurus and Orion, then the inferred masses of the X-ray detected TTS are $M_{*} \lesssim 0.7 M_{\odot}$. Although this result is based only on partial X-ray coverage of the L1228 dark cloud, it is nevertheless consistent with the broader picture that L1228 has formed only low-mass stars.

The scientific results reported in this article are based in part on observations made by the Chandra X-ray Observatory. We thank Kimberly Sokal and Bryan Nagel for assistance with data reduction. This work is based in part on archival data obtained with the Spitzer Space Telescope, which is operated by the Jet Propulsion Laboratory (JPL), California Institute of Technology (Caltech) under a contract with NASA. This publication makes use of data products from the Wide-field Infrared Survey Explorer (WISE), which is a joint project of the University of California, Los Angeles, and JPL/Caltech, funded by NASA. This research has made use of data products from the Two Micron All-Sky Survey (2MASS), which is a joint project of the University of Massachusetts and the Infrared Processing and Analysis Center (IPAC), funded by NASA and the National Science Foundation. These data were served by the NASA/IPAC Infrared Science Archive, which is operated by JPL/Caltech under contract with NASA.

\section{REFERENCES}

Anders, E., \& Grevesse, N. 1989, GeCoA, 53, 197

Bally, J., Devine, D., Fesen, R. A., \& Lane, A. P. 1995, ApJ, 454, 345

Brandt, W. N., Alexander, D. M., Hornschemeier, A. E., et al. 2001, AJ, 122,2810

Chapman, N. L., \& Mundy, L. G. 2009, ApJ, 699, 1866

Cowie, L. L., Garmire, G. P., Bautz, M. W., et al. 2002, ApJL, 566, L5 Devine, D., Bally, J., Chiriboga, D., \& Smart, K. 2009, AJ, 137, 3993

Evans, N. J., Allen, L. E., Blake, G. A., et al. 2003, PASP, 115, 965

Fazio, G., Hora, J. L., Allen, L. E., et al. 2004, ApJS, 154, 10

Gorenstein, P. 1975, ApJ, 198, 95

Gregory, P. C., \& Loredo, T. J. 1992, ApJ, 398, 146

Gregory, P. C., \& Loredo, T. J. 1996, ApJ, 473, 1059

Grenier, I. A., Lebrun, F., Arnaud, M., Dame, T. M., \& Thaddeus, P. 1989, ApJ, 347,231

Gutermuth, R. A., Myers, P. C., Megeath, S. T., et al. 2008, ApJ, 674, 336

Haisch, K. E., Lada, E. A., Pina, R. K., Telesco, C. M., \& Lada, C. L. 2001, AJ, 121,1512

Imanishi, K., Koyama, K., \& Tsuboi, Y. 2001, ApJ, 557, 747

Kirk, J. M., Ward-Thompson, D., Di Francesco, J., et al. 2009, ApJS, 185, 198

Kiss, Z. T., Tóth, L. V., Krause, O., Kun, M., \& Stickel, M. 2006, A\&A, 453, 923

Koenig, X., Leisawitz, D. T., Benford, D. J., et al. 2012, ApJ, 744, 130

Kun, M. 1998, ApJS, 115, 59

Kun, M., Balog, Z., Kenyon, S. J., Mamajek, E. E., \& Gutermuth, R. A. 2009, ApJS, 185, 451

Kun, M., Kiss, Z. T., \& Balog, Z. 2008, in Handbook of Star Forming Regions: Volume 1, The Northern Sky, ed. B. Reipurth (ASP Monograph Publications; San Francisco, CA: ASP), 136

Lynds, B. T. 1962, ApJS, 7, 1

Makovoz, D., \& Marleau, F. 2005, PASP, 117, 1113

Ogura, K., \& Sato, F. 1990, PASJ, 42, 583

Olano, C. A., Meschin, P. I., \& Niemela, V. S. 2006, MNRAS, 369, 867

Preibisch, T., Kim, Y.-C., Favata, F., et al. 2005, ApJS, 160, 401 
Rebull, L., Koenig, X. P., Padgett, D. L., et al. 2011, ApJS, 196, 4

Rebull, L., Padgett, D. L., McCabe, C.-E., et al. 2010, ApJS, 186, 259

Reipurth, B., Rodríguez, L. F., Anglada, G., \& Bally, J. 2004, AJ, 127, 1736

Rieke, G., Young, E. T., Engelbracht, C. W., et al. 2004, ApJS, 154, 25

Robitaille, T. P., Whitney, B. A., Indebetouw, R., \& Wood, K. 2007, ApJS, 169,328
Skrutskie, M., Cutri, R. M., Stiening, R., et al. 2006, AJ, 131, 1163

Tachihara, K., Neuhäuser, R., Kun, M., \& Fukui, Y. 2005, A\&A, 437, 919

Telleschi, A., Güdel, M., Briggs, K. R., Audard, M., \& Palla, F. 2007, A\&A, 468,425

Vuong, M. H., Montmerle, T., Grosso, N., et al. 2003, A\&A, 408, 581

Werner, M., Roellig, T. L., Low, F. J., et al. 2004, ApJS, 154, 1

Wright, E., Eisenhardt, P. R. M., Mainzer, A. K., et al. 2010, AJ, 140, 1868 